\title{
NUMERICAL DISSIPATION SWITCH FOR TWO-DIMENSIONAL CENTRAL-UPWIND SCHEMES
}

\author{
Alexander Kurganov ${ }^{1,2}$, Yongle LiU ${ }^{3,1, *}$ And Vladimir Zeitlin ${ }^{4,1}$
}

\begin{abstract}
We propose a numerical dissipation switch, which helps to control the amount of numerical dissipation present in central-upwind schemes. Our main goal is to reduce the numerical dissipation without risking oscillations. This goal is achieved with the help of a more accurate estimate of the local propagation speeds in the parts of the computational domain, which are near contact discontinuities and shears. To this end, we introduce a switch parameter, which depends on the distributions of energy in the $x$ - and $y$-directions. The resulting new central-upwind is tested on a number of numerical examples, which demonstrate the superiority of the proposed method over the original central-upwind scheme.
\end{abstract}

Mathematics Subject Classification. 76M12, 65M08, 35L65, 86-08.

Received June 1, 2020. Accepted February 17, 2021.

\section{INTRODUCTION}

We consider two-dimensional (2-D) hyperbolic systems of conservation laws, which can be written as

$$
\boldsymbol{U}_{t}+\boldsymbol{F}(\boldsymbol{U})_{x}+\boldsymbol{G}(\boldsymbol{U})_{y}=\mathbf{0},
$$

where $x$ and $y$ are spatial variables, $t$ is time, $\boldsymbol{U}(x, y, t) \in \mathbb{R}^{d}$ is the vector of unknowns, and $\boldsymbol{F}$ and $\boldsymbol{G}$ are the $x$ - and $y$-fluxes, respectively.

It is well-known that numerically computing solutions of (1.1) is a challenging task since many of these solutions develop very complicated nonsmooth structures including shock waves, contact discontinuities and rarefaction zones. One of the most popular numerical tools for hyperbolic systems of conservation and balance laws are finite-volume methods; see, e.g., [9, 16, 18-20,33,51]. Most of the finite-volume methods are designed for the one-dimensional (1-D) version of (1.1). The classical Godunov scheme [10] is based on a global piecewise constant approximation of the computed solution combined with its upwind evolution in time. The latter requires exact solution of the Riemann problems arising at every cell interface in the beginning of each time step. Riemann

Keywords and phrases. Central-upwind schemes, numerical dissipation, local speeds of propagation, Euler equations of gas dynamics, thermal rotating shallow water equations.

1 Department of Mathematics, Southern University of Science and Technology, Shenzhen 518055, P.R. China.

2 SUSTech International Center for Mathematics, Southern University of Science and Technology, Shenzhen 518055, P.R. China.

3 Department of Mathematics, Harbin Institute of Technology, Harbin 150001, P.R. China.

4 Laboratoire de Météorologie Dynamique, Sorbonne Université (SU), Ecole Normale Supérieure (ENS), CNRS, Paris 75231, France.

*Corresponding author: liuyl2017@mail.sustech.edu.cn 
problems are, however, quite hard (sometimes impossible) and time-consuming to solve, and therefore many approximate Riemann problem solvers have been proposed since 1980s. For instance, the celebrated Roe solver [45] is based on the local linearizations of the flux Jacobian followed by the exact solution of the Riemann problems for the linearized systems. The Roe solver often provides a very sharp resolution of the complicated solution structure including the linearly degenerate contact waves, which are especially challenging to accurately capture. The drawback of the Roe solver is a lack of robustness as the linearized systems may, in some situations, be a poor approximation of the original nonlinear system. An alternative, much more robust Harten-Lax-van Leer (HLL) approximate Riemann problem solver was proposed in [15]. The drawback of the HLL solver is related to the fact that it is based on the fastest and slowest shock/rarefaction waves and thus may leads to excessive smearing of contact discontinuities. For other Riemann problem solvers we refer the reader to [51] and references therein. The 1-D finite-volume methods can be extended to the higher-dimensional cases in a dimension-bydimension manner. There are also genuinely multidimensional methods (see, e.g., $[2,21,22,25,31,32,40]$ ), which are often advantageous as they are less prone to grid effects.

In this paper, we focus on genuinely multidimensional central-upwind schemes, which are Riemann-problemsolver-free methods that can be applied to a wide variety of hyperbolic systems of conservation and balance laws as a robust, stable and highly accurate "black-box" solver. Central-upwind schemes were originally developed in [23-25] as a low dissipative alternative to staggered central schemes - the second-order Nessyahu-Tadmor scheme [42] and its multidimensional and higher-order extensions (see, e.g., [1,17,34-36]). The amount of numerical dissipation present in central-upwind scheme was reduced in $[21,26]$. Our goal is to further reduce the numerical dissipation by introducing a numerical dissipation switch, which is similar to the switch recently developed in [43]. We present the switch in the context of Euler equations of gas dynamics. It can be, however, used in many other models as, for instance, thermal rotating shallow water equations as it is done in Example 8 below.

The paper is organized as follows. In Section 2, we briefly review the semi-discrete central-upwind scheme from [5,21]. In Section 3, we discuss application of the scheme to the 2-D Euler equations of gas dynamics and present the numerical dissipation switch. In Section 4, we demonstrate the effects of the switch on a number of numerical examples.

\section{SEMi-DisCREte CENTRAL-UPWIND SCHEME - BRIEF OVERVIEW}

In this section, we briefly review the 2-D semi-discrete second-order central-upwind scheme from [5,21].

We divide the computational domain into a set of uniform Cartesian cells $C_{j, k}:=\left[x_{j-\frac{1}{2}}, x_{j+\frac{1}{2}}\right] \times\left[y_{k-\frac{1}{2}}, y_{k+\frac{1}{2}}\right]$, which are centered at $\left(x_{j}, y_{k}\right)=\left(x_{j-\frac{1}{2}}+\Delta x / 2, y_{k-\frac{1}{2}}+\Delta y / 2\right)$. We assume that the solution of (1.1), realized in terms of its cell averages,

$$
\overline{\boldsymbol{U}}_{j, k}(t): \approx \frac{1}{\Delta x \Delta y} \iint_{C_{j, k}} \boldsymbol{U}(x, y, t) \mathrm{d} x \mathrm{~d} y,
$$

is available at a certain time $t$. The solution is then evolved in time by solving the following system of ODEs:

$$
\frac{\mathrm{d}}{\mathrm{d} t} \overline{\boldsymbol{U}}_{j, k}(t)=-\frac{\mathcal{F}_{j+\frac{1}{2}, k}(t)-\mathcal{F}_{j-\frac{1}{2}, k}(t)}{\Delta x}-\frac{\mathcal{G}_{j, k+\frac{1}{2}}(t)-\mathcal{G}_{j, k-\frac{1}{2}}(t)}{\Delta y},
$$

where $\mathcal{F}_{j+\frac{1}{2}, k}(t)$ and $\mathcal{G}_{j, k+\frac{1}{2}}(t)$ are the central-upwind numerical fluxes in the $x$ - and $y$-directions, respectively.

Remark 2.1. For the sake of brevity, we will omit the dependence of all of the indexed finite-volume quantities on $t$ in the rest of this paper. 
The central-upwind numerical fluxes from [21] are

$$
\begin{gathered}
\mathcal{F}_{j+\frac{1}{2}, k}=\frac{a_{j+\frac{1}{2}, k}^{+} \boldsymbol{F}\left(\boldsymbol{U}_{j, k}^{\mathrm{E}}\right)-a_{j+\frac{1}{2}, k}^{-} \boldsymbol{F}\left(\boldsymbol{U}_{j+1, k}^{\mathrm{W}}\right)}{a_{j+\frac{1}{2}, k}^{+}-a_{j+\frac{1}{2}, k}^{-}}+\frac{a_{j+\frac{1}{2}, k}^{+} a_{j+\frac{1}{2}, k}^{-}}{a_{j+\frac{1}{2}, k}^{+}-a_{j+\frac{1}{2}, k}^{-}}\left[\boldsymbol{U}_{j+1, k}^{\mathrm{W}}-\boldsymbol{U}_{j, k}^{\mathrm{E}}-\delta \boldsymbol{U}_{j+\frac{1}{2}, k}\right], \\
\mathcal{G}_{j, k+\frac{1}{2}}=\frac{b_{j, k+\frac{1}{2}}^{+} \boldsymbol{G}\left(\boldsymbol{U}_{j, k}^{\mathrm{N}}\right)-b_{j, k+\frac{1}{2}}^{-} \boldsymbol{G}\left(\boldsymbol{U}_{j, k+1}^{\mathrm{S}}\right)}{b_{j, k+\frac{1}{2}}^{+}-b_{j, k+\frac{1}{2}}^{-}}+\frac{b_{j, k+\frac{1}{2}}^{+} b_{j, k+\frac{1}{2}}^{-}}{b_{j, k+\frac{1}{2}}^{+}-b_{j, k+\frac{1}{2}}^{-}}\left[\boldsymbol{U}_{j, k+1}^{\mathrm{S}}-\boldsymbol{U}_{j, k}^{\mathrm{N}}-\delta \boldsymbol{U}_{j, k+\frac{1}{2}}\right] .
\end{gathered}
$$

Here, $\delta \boldsymbol{U}_{j+\frac{1}{2}, k}$ and $\delta \boldsymbol{U}_{j, k+\frac{1}{2}}$ are built-in "anti-diffusion" terms:

$$
\begin{aligned}
& \delta \boldsymbol{U}_{j+\frac{1}{2}, k}=\operatorname{minmod}\left(\boldsymbol{U}_{j+1, k}^{\mathrm{SW}}-\boldsymbol{U}_{j+\frac{1}{2}, k}^{*}, \boldsymbol{U}_{j+\frac{1}{2}, k}^{*}-\boldsymbol{U}_{j, k}^{\mathrm{SE}}, \boldsymbol{U}_{j+1, k}^{\mathrm{NW}}-\boldsymbol{U}_{j+\frac{1}{2}, k}^{*}, \boldsymbol{U}_{j+\frac{1}{2}, k}^{*}-\boldsymbol{U}_{j, k}^{\mathrm{NE}}\right), \\
& \delta \boldsymbol{U}_{j, k+\frac{1}{2}}=\operatorname{minmod}\left(\boldsymbol{U}_{j, k+1}^{\mathrm{SW}}-\boldsymbol{U}_{j, k+\frac{1}{2}}^{*}, \boldsymbol{U}_{j, k+\frac{1}{2}}^{*}-\boldsymbol{U}_{j, k}^{\mathrm{NW}}, \boldsymbol{U}_{j, k+1}^{\mathrm{SE}}-\boldsymbol{U}_{j, k+\frac{1}{2}}^{*}, \boldsymbol{U}_{j, k+\frac{1}{2}}^{*}-\boldsymbol{U}_{j, k}^{\mathrm{NE}}\right),
\end{aligned}
$$

where

$$
\begin{gathered}
\boldsymbol{U}_{j+\frac{1}{2}, k}^{*}=\frac{a_{j+\frac{1}{2}, k}^{+} \boldsymbol{U}_{j+1, k}^{\mathrm{W}}-a_{j+\frac{1}{2}, k}^{-} \boldsymbol{U}_{j, k}^{\mathrm{E}}-\left\{\boldsymbol{F}\left(\boldsymbol{U}_{j+1, k}^{\mathrm{W}}\right)-\boldsymbol{F}\left(\boldsymbol{U}_{j, k}^{\mathrm{E}}\right)\right\}}{a_{j+\frac{1}{2}, k}^{+}-a_{j+\frac{1}{2}, k}^{-}}, \\
\boldsymbol{U}_{j, k+\frac{1}{2}}^{*}=\frac{b_{j, k+\frac{1}{2}}^{+} \boldsymbol{U}_{j, k+1}^{\mathrm{S}}-b_{j, k+\frac{1}{2}}^{-} \boldsymbol{U}_{j, k}^{\mathrm{N}}-\left\{\boldsymbol{G}\left(\boldsymbol{U}_{j, k+1}^{\mathrm{S}}\right)-\boldsymbol{G}\left(\boldsymbol{U}_{j, k}^{\mathrm{N}}\right)\right\}}{b_{j, k+\frac{1}{2}}^{+}-b_{j, k+\frac{1}{2}}^{-}} .
\end{gathered}
$$

In (2.2)-(2.4), the point values

$$
\begin{aligned}
& \boldsymbol{U}_{j, k}^{\mathrm{E}}=\overline{\boldsymbol{U}}_{j, k}+\frac{\Delta x}{2}\left(\boldsymbol{U}_{x}\right)_{j, k}, \quad \boldsymbol{U}_{j, k}^{\mathrm{W}}=\overline{\boldsymbol{U}}_{j, k}-\frac{\Delta x}{2}\left(\boldsymbol{U}_{x}\right)_{j, k}, \\
& \boldsymbol{U}_{j, k}^{\mathrm{N}}=\overline{\boldsymbol{U}}_{j, k}+\frac{\Delta y}{2}\left(\boldsymbol{U}_{y}\right)_{j, k}, \quad \boldsymbol{U}_{j, k}^{\mathrm{S}}=\overline{\boldsymbol{U}}_{j, k}-\frac{\Delta y}{2}\left(\boldsymbol{U}_{y}\right)_{j, k}
\end{aligned}
$$

and

$$
\boldsymbol{U}_{j, k}^{\mathrm{NE}(\mathrm{NW})}=\overline{\boldsymbol{U}}_{j, k} \pm \frac{\Delta x}{2}\left(\boldsymbol{U}_{x}\right)_{j, k}+\frac{\Delta y}{2}\left(\boldsymbol{U}_{y}\right)_{j, k}, \quad \boldsymbol{U}_{j, k}^{\mathrm{SE}(\mathrm{SW})}=\overline{\boldsymbol{U}}_{j, k} \pm \frac{\Delta x}{2}\left(\boldsymbol{U}_{x}\right)_{j, k}-\frac{\Delta y}{2}\left(\boldsymbol{U}_{y}\right)_{j, k}
$$

are to be computed using a nonlinear limiter to minimize spurious oscillations. For example, one can use the generalized minmod limiter (see, e.g., [37,42,50,52]):

$$
\begin{aligned}
& \left(\boldsymbol{U}_{x}\right)_{j, k}=\operatorname{minmod}\left(\sigma \frac{\overline{\boldsymbol{U}}_{j, k}-\overline{\boldsymbol{U}}_{j-1, k}}{\Delta x}, \frac{\overline{\boldsymbol{U}}_{j+1, k}-\overline{\boldsymbol{U}}_{j-1, k}}{2 \Delta x}, \sigma \frac{\overline{\boldsymbol{U}}_{j+1, k}-\overline{\boldsymbol{U}}_{j, k}}{\Delta x}\right), \\
& \left(\boldsymbol{U}_{y}\right)_{j, k}=\operatorname{minmod}\left(\sigma \frac{\overline{\boldsymbol{U}}_{j, k}-\overline{\boldsymbol{U}}_{j, k-1}}{\Delta y}, \frac{\overline{\boldsymbol{U}}_{j, k+1}-\overline{\boldsymbol{U}}_{j, k-1}}{2 \Delta y}, \sigma \frac{\overline{\boldsymbol{U}}_{j, k+1}-\overline{\boldsymbol{U}}_{j, k}}{\Delta y}\right),
\end{aligned}
$$

where the parameter $\sigma \in[1,2]$ helps to control the smoothness of the reconstructed values (larger values of $\sigma$ correspond to sharper, but more oscillatory reconstructions).

The minmod function used in (2.3) and (2.7) is defined by

$$
\operatorname{minmod}\left(z_{1}, z_{2}, \ldots\right):= \begin{cases}\min _{k}\left\{z_{k}\right\}, & \text { if } z_{k}>0 \forall k, \\ \max _{k}\left\{z_{k}\right\}, & \text { if } z_{k}<0 \forall k, \\ 0, & \text { otherwise }\end{cases}
$$

and is applied in both (2.3) and (2.7) in a component-wise manner. 

by

Finally, $a_{j+\frac{1}{2}, k}^{ \pm}$and $b_{j, k+\frac{1}{2}}^{ \pm}$are the one-sided local propagation speeds, which can be estimated, for example,

$$
\begin{aligned}
& a_{j+\frac{1}{2}, k}^{+}=\max \left\{\lambda_{d}\left(\frac{\partial \boldsymbol{F}}{\partial \boldsymbol{U}}\left(\boldsymbol{U}_{j+1, k}^{\mathrm{W}}\right)\right), \lambda_{d}\left(\frac{\partial \boldsymbol{F}}{\partial \boldsymbol{U}}\left(\boldsymbol{U}_{j, k}^{\mathrm{E}}\right)\right), 0\right\}, \\
& a_{j+\frac{1}{2}, k}^{-}=\min \left\{\lambda_{1}\left(\frac{\partial \boldsymbol{F}}{\partial \boldsymbol{U}}\left(\boldsymbol{U}_{j+1, k}^{\mathrm{W}}\right)\right), \lambda_{1}\left(\frac{\partial \boldsymbol{F}}{\partial \boldsymbol{U}}\left(\boldsymbol{U}_{j, k}^{\mathrm{E}}\right)\right), 0\right\}, \\
& b_{j, k+\frac{1}{2}}^{+}=\max \left\{\lambda_{d}\left(\frac{\partial \boldsymbol{G}}{\partial \boldsymbol{U}}\left(\boldsymbol{U}_{j, k+1}^{\mathrm{S}}\right)\right), \lambda_{d}\left(\frac{\partial \boldsymbol{G}}{\partial \boldsymbol{U}}\left(\boldsymbol{U}_{j, k}^{\mathrm{N}}\right)\right), 0\right\}, \\
& b_{j, k+\frac{1}{2}}^{-}=\min \left\{\lambda_{1}\left(\frac{\partial \boldsymbol{G}}{\partial \boldsymbol{U}}\left(\boldsymbol{U}_{j, k+1}^{\mathrm{S}}\right)\right), \lambda_{1}\left(\frac{\partial \boldsymbol{G}}{\partial \boldsymbol{U}}\left(\boldsymbol{U}_{j, k}^{\mathrm{N}}\right)\right), 0\right\},
\end{aligned}
$$

where $\lambda_{1}<\lambda_{2}<\ldots<\lambda_{d}$ are the $d$ eigenvalues of the corresponding Jacobians, $\frac{\partial \boldsymbol{F}}{\partial \boldsymbol{U}}$ and $\frac{\partial \boldsymbol{G}}{\partial \boldsymbol{U}}$.

Remark 2.2. We would like to point out that the upper bounds on the local speeds of propagation given in (2.8) may be inaccurate; see, for example, [14]. Moreover, we have implemented the strategy from [14] in Examples 1-6 below, but since it did not make any impact on the results in any of the conducted numerical experiments, we only use the straightforward estimates (2.8) in this paper.

Remark 2.3. We note that the semi-discrete central-upwind scheme (2.1)-(2.8) is a system of time dependent ODEs, which should be integrated by a sufficiently accurate, efficient and stable ODE solver. In our numerical experiments, we have used the three-stage third-order strong stability preserving (SSP) Runge-Kutta method (see, e.g., $[11,12]$ ) with an adaptive time step computed at every time level using the CFL number 0.475:

$$
\Delta t=0.475 \min \left\{\frac{\Delta x}{a_{\max }}, \frac{\Delta y}{b_{\max }}\right\}
$$

where

$$
a_{\max }:=\max _{j, k}\left\{a_{j+\frac{1}{2}, k}^{+},-a_{j+\frac{1}{2}, k}^{-}\right\}, \quad b_{\max }:=\max _{j, k}\left\{b_{j, k+\frac{1}{2}}^{+},-b_{j, k+\frac{1}{2}}^{-}\right\} .
$$

\section{NUMERICAL DisSiPATION SWITCH}

In this section, we present a way to reduce the amount of numerical dissipation present in the central-upwind scheme from Section 2 applied to the 2-D Euler equations of gas dynamics:

$$
\begin{aligned}
\rho_{t}+(\rho u)_{x}+(\rho v)_{y} & =0, \\
(\rho u)_{t}+\left(\rho u^{2}+p\right)_{x}+(\rho u v)_{y} & =0, \\
(\rho v)_{t}+(\rho u v)_{x}+\left(\rho v^{2}+p\right)_{y} & =0, \\
E_{t}+[u(E+p)]_{x}+[v(E+p)]_{y} & =0 .
\end{aligned}
$$

Here, $\rho$ is the density, $u$ and $v$ are the $x$-and $y$-velocities, respectively, $p$ is the pressure, and $E$ is the total energy, which is related to the primitive variables $\rho, u, v$ and $p$ via the equation of state (EOS). For the ideal gas, the EOS is

$$
E=\frac{p}{\gamma-1}+\frac{\rho}{2}\left(u^{2}+v^{2}\right)
$$

where $\gamma$ is the specific heat ratio taken to be 1.4 in all of the numerical examples reported in Section 4 .

According to (2.8), the one-sided local propagation speeds can be estimated by

$$
\begin{aligned}
a_{j+\frac{1}{2}, k}^{+} & =\max \left\{u_{j, k}^{\mathrm{E}}+c_{j, k}^{\mathrm{E}}, u_{j+1, k}^{\mathrm{W}}+c_{j+1, k}^{\mathrm{W}}, 0\right\}, \\
a_{j+\frac{1}{2}, k}^{-} & =\min \left\{u_{j, k}^{\mathrm{E}}-c_{j, k}^{\mathrm{E}}, u_{j+1, k}^{\mathrm{W}}-c_{j+1, k}^{\mathrm{W}}, 0\right\}, \\
b_{j, k+\frac{1}{2}}^{+} & =\max \left\{v_{j, k}^{\mathrm{N}}+c_{j, k}^{\mathrm{N}}, v_{j, k+1}^{\mathrm{S}}+c_{j, k+1}^{\mathrm{S}}, 0\right\}, \\
b_{j, k+\frac{1}{2}}^{-} & =\min \left\{v_{j, k}^{\mathrm{N}}-c_{j, k}^{\mathrm{N}}, v_{j, k+1}^{\mathrm{S}}-c_{j, k+1}^{\mathrm{S}}, 0\right\},
\end{aligned}
$$


where $c:=\sqrt{\gamma p / \rho}$ is the speed of sound, and

$$
\begin{aligned}
u_{j, k}^{\mathrm{E}} & =\frac{(\overline{\rho u})_{j, k}^{\mathrm{E}}}{\bar{\rho}_{j, k}^{\mathrm{E}}}, \quad u_{j+1, k}^{\mathrm{W}}=\frac{(\overline{\rho u})_{j+1, k}^{\mathrm{W}}}{\bar{\rho}_{j+1, k}^{\mathrm{W}}}, \quad u_{j, k}^{\mathrm{N}}=\frac{(\overline{\rho u})_{j, k}^{\mathrm{N}}}{\bar{\rho}_{j, k}^{\mathrm{N}}}, \quad u_{j, k+1}^{\mathrm{S}}=\frac{(\overline{\rho u})_{j, k+1}^{\mathrm{S}}}{\bar{\rho}_{j, k+1}^{\mathrm{S}}}, \\
v_{j, k}^{\mathrm{E}} & =\frac{(\overline{\rho v})_{j, k}^{\mathrm{E}}}{\bar{\rho}_{j, k}^{\mathrm{E}}}, \quad v_{j+1, k}^{\mathrm{W}}=\frac{(\overline{\rho v})_{j+1, k}^{\mathrm{W}}}{\bar{\rho}_{j+1, k}^{\mathrm{W}}}, \quad v_{j, k}^{\mathrm{N}}=\frac{(\overline{\rho v})_{j, k}^{\mathrm{N}}}{\bar{\rho}_{j, k}^{\mathrm{N}}}, \quad v_{j, k+1}^{\mathrm{S}}=\frac{(\overline{\rho v})_{j, k+1}^{\mathrm{S}}}{\bar{\rho}_{j, k+1}^{\mathrm{S}}}, \\
p_{j, k}^{\mathrm{E}} & =(\gamma-1)\left[\bar{E}_{j, k}^{\mathrm{E}}-\frac{\bar{\rho}_{j, k}^{\mathrm{E}}}{2}\left(\left(u_{j, k}^{\mathrm{E}}\right)^{2}+\left(v_{j, k}^{\mathrm{E}}\right)^{2}\right)\right], \\
p_{j+1, k}^{\mathrm{W}} & =(\gamma-1)\left[\bar{E}_{j+1, k}^{\mathrm{W}}-\frac{\bar{\rho}_{j+1, k}^{\mathrm{W}}}{2}\left(\left(u_{j+1, k}^{\mathrm{W}}\right)^{2}+\left(v_{j+1, k}^{\mathrm{W}}\right)^{2}\right)\right], \\
p_{j, k}^{\mathrm{N}} & =(\gamma-1)\left[\bar{E}_{j, k}^{\mathrm{N}}-\frac{\bar{\rho}_{j, k}^{\mathrm{N}}}{2}\left(\left(u_{j, k}^{\mathrm{N}}\right)^{2}+\left(v_{j, k}^{\mathrm{N}}\right)^{2}\right)\right], \\
p_{j, k+1}^{\mathrm{S}} & =(\gamma-1)\left[\bar{E}_{j, k+1}^{\mathrm{S}}-\frac{\bar{\rho}_{j, k+1}^{\mathrm{S}}\left(\left(u_{j, k+1}^{\mathrm{S}}\right)^{2}+\left(v_{j, k+1}^{\mathrm{S}}\right)\right],}{2}, \quad c_{j, k}^{\mathrm{N}}=\sqrt{\frac{\gamma p_{j, k}^{\mathrm{N}}}{\bar{\rho}_{j, k}^{\mathrm{N}}}}, \quad c_{j, k+1}^{\mathrm{S}}=\sqrt{\frac{\gamma p_{j, k+1}^{\mathrm{S}}}{\bar{\rho}_{j, k+1}^{\mathrm{S}}}} .\right.
\end{aligned}
$$

The estimate (3.2), however, may be very inaccurate in certain situations. In order to illustrate this, let us consider a particular example with the initial data that correspond to a stationary shear flow. Let us set up the discrete initial data in such a way that the boundary between the two parts of the fluid moving in the positive and negative $y$-directions is located at $x=x_{J+\frac{1}{2}}$ for a certain index $J$; see Figure 1 . One can easily verify that for this data setting

$$
\mathcal{G}_{j, k+\frac{1}{2}}-\mathcal{G}_{j, k-\frac{1}{2}}=0, \quad \forall(j, k), \quad \mathcal{F}_{j+\frac{1}{2}, k}-\mathcal{F}_{j+\frac{1}{2}, k}=0, \quad \forall j \neq J \text { and } j \neq J+1, \forall k .
$$

Let us compute $\mathcal{F}_{J-\frac{1}{2}, k}, \mathcal{F}_{J+\frac{1}{2}, k}$ and $\mathcal{F}_{J+\frac{3}{2}, k}$. To this end, we first use (3.2) to obtain the corresponding one-sided local speeds of propagation:

$$
a_{J-\frac{1}{2}, k}^{-}=a_{J-\frac{1}{2}, k}^{+}-=a_{J+\frac{1}{2}, k}^{-}=-2, \quad a_{J+\frac{1}{2}, k}^{+}=a_{J-\frac{3}{2}, k}^{-}=a_{J-\frac{3}{2}, k}^{+}-=1, \quad \forall k,
$$

and then use them to compute the numerical fluxes, which are

$$
\mathcal{F}_{J-\frac{1}{2}, k}=(0,1,0,0)^{\top}, \quad \mathcal{F}_{J+\frac{1}{2}, k}=(-7 / 15,1,14 / 15,0)^{\top}, \quad \mathcal{F}_{J+\frac{3}{2}, k}=(0,1,0,0)^{\top} .
$$

As one can see, the initial equilibrium will not be preserved at the discrete level as $\bar{\rho}_{J}, \bar{\rho}_{J+1},(\overline{\rho v})_{J}$ and $(\overline{\rho v})_{J+1}$ will change after the first time step. We note that this occurs precisely because of the discrepancy between $a_{J+\frac{1}{2}, k}^{-}$and $a_{J+\frac{1}{2}, k}^{+}$values caused by a very inaccurate overestimate of the local speeds, which, in turn, leads to excessive numerical dissipation in the shear area.

Therefore, in order to improve the resolution of the central-upwind scheme, we develop a numerical dissipation switch, which will help to better estimate the one-sided local propagation speeds by reducing the magnitudes of $a_{j+\frac{1}{2}, k}^{ \pm}$and/or $b_{j, k+\frac{1}{2}}^{ \pm}$in the areas where the solution is near contact discontinuities and shears. By doing this, we will reduce the size of the control volumes used for the evolution of the solution in those areas (see [21] for details on the derivation of central-upwind schemes via the integral form of conservation laws) and thus reduce the amount of numerical dissipation there. At the same time, we will keep a sufficient amount of numerical 


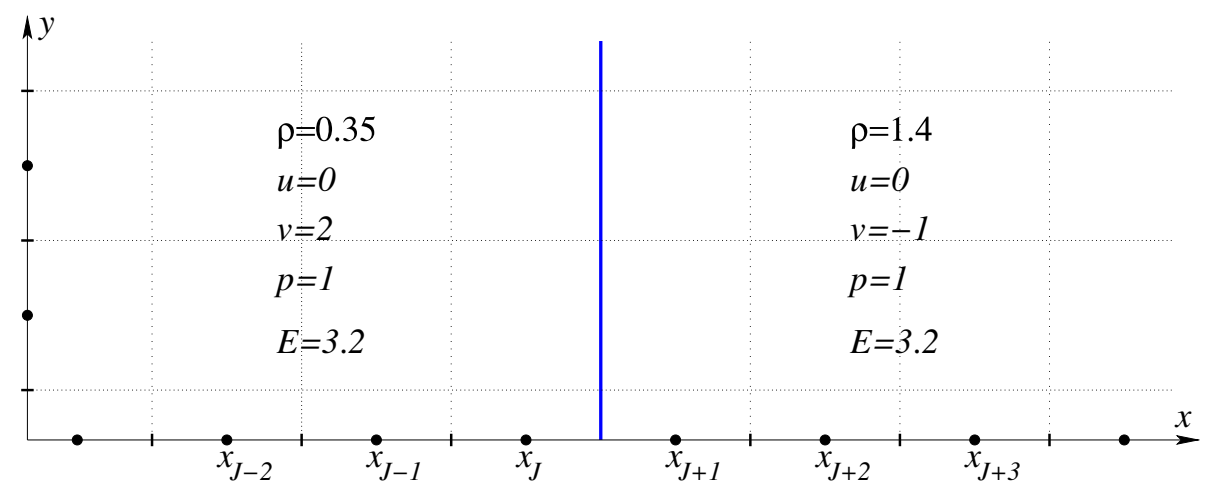

FiguRE 1. Initial setting for a stationary shear flow.

dissipation near shock waves to guarantee the stability of the method. To this end, we follow the approach in [43] and add a switch on the sound speed parts of the estimates in (3.2), which are replaced by

$$
\begin{aligned}
a_{j+\frac{1}{2}, k}^{+} & =\max \left\{u_{j, k}^{\mathrm{E}}+\alpha_{j+\frac{1}{2}, k} c_{j, k}^{\mathrm{E}}, u_{j+1, k}^{\mathrm{W}}+\alpha_{j+\frac{1}{2}, k} c_{j+1, k}^{\mathrm{W}}, 0\right\}, \\
a_{j+\frac{1}{2}, k}^{-} & =\min \left\{u_{j, k}^{\mathrm{E}}-\alpha_{j+\frac{1}{2}, k} c_{j, k}^{\mathrm{E}}, u_{j+1, k}^{\mathrm{W}}-\alpha_{j+\frac{1}{2}, k} c_{j+1, k}^{\mathrm{W}}, 0\right\}, \\
b_{j, k+\frac{1}{2}}^{+} & =\max \left\{v_{j, k}^{\mathrm{N}}+\beta_{j, k+\frac{1}{2}} c_{j, k}^{\mathrm{N}}, v_{j, k+1}^{\mathrm{S}}+\beta_{j, k+\frac{1}{2}} c_{j, k+1}^{\mathrm{S}}, 0\right\}, \\
b_{j, k+\frac{1}{2}}^{-} & =\min \left\{v_{j, k}^{\mathrm{N}}-\beta_{j, k+\frac{1}{2}} c_{j, k}^{\mathrm{N}}, v_{j, k+1}^{\mathrm{S}}-\beta_{j, k+\frac{1}{2}} c_{j, k+1}^{\mathrm{S}}, 0\right\},
\end{aligned}
$$

where $\alpha_{j+\frac{1}{2}, k} \in[0,1]$ and $\beta_{j, k+\frac{1}{2}} \in[0,1]$ are the switch parameters designed to be small near contact discontinuities and shears. This goal is achieved by setting

$$
\alpha_{j+\frac{1}{2}, k}= \begin{cases}\frac{\Delta \alpha_{j+\frac{1}{2}, k}^{(1)},}{\Delta \alpha_{j+\frac{1}{2}, k}} & \text { if } \Delta \alpha_{j+\frac{1}{2}, k}>\varepsilon \\ 0, & \text { otherwise }\end{cases}
$$

where

$$
\begin{aligned}
& \Delta \alpha_{j+\frac{1}{2}, k}^{(1)}=\left|\frac{p_{j+1, k}^{\mathrm{W}}-p_{j, k}^{\mathrm{E}}}{\gamma-1}+\frac{\rho_{j+1, k}^{\mathrm{W}}\left(u_{j+1, k}^{\mathrm{W}}\right)^{2}-\rho_{j, k}^{\mathrm{E}}\left(u_{j, k}^{\mathrm{E}}\right)^{2}}{2}\right|, \\
& \Delta \alpha_{j+\frac{1}{2}, k}^{(2)}=\left|\frac{\rho_{j+1, k}^{\mathrm{W}}\left(v_{j+1, k}^{\mathrm{W}}\right)^{2}-\rho_{j, k}^{\mathrm{E}}\left(v_{j, k}^{\mathrm{E}}\right)^{2}}{2}\right|, \quad \Delta \alpha_{j+\frac{1}{2}, k}=\sqrt{\left(\Delta \alpha_{j+\frac{1}{2}, k}^{(1)}\right)^{2}+\left(\Delta \alpha_{j+\frac{1}{2}, k}^{(2)}\right)^{2}} .
\end{aligned}
$$

We note that equations (3.4), (3.5) rely on comparing difference quotients of the $x$-momentum fluxes normal to the boundary between cells $C_{j, k}$ and $C_{j+1, k}$ with those of the shear-flux tangential to the same boundary. The motivation behind using (3.3)-(3.5) is based on the observation that in the case when $\alpha_{j+\frac{1}{2}, k}^{(1)}$ is much smaller than $\alpha_{j+\frac{1}{2}, k}^{(2)}$, the local speed of propagation in the $x$-direction at the boundary between cells $C_{j, k}$ and $C_{j+1, k}$ is determined by the intermediate eigenvalue $u$ rather than by $u+c$ and $u-c$. 
Similarly, the switch in the $y$-direction is constructed using

$$
\beta_{j, k+\frac{1}{2}}= \begin{cases}\frac{\Delta \beta_{j, k+\frac{1}{2}}^{(1)}}{\Delta \beta_{j, k+\frac{1}{2}}}, & \text { if } \Delta \beta_{j, k+\frac{1}{2}}>\varepsilon \\ 0, & \text { otherwise }\end{cases}
$$

where

$$
\begin{aligned}
& \Delta \beta_{j, k+\frac{1}{2}}^{(1)}=\left|\frac{p_{j, k+1}^{\mathrm{S}}-p_{j, k}^{\mathrm{N}}}{\gamma-1}+\frac{\rho_{j, k+1}^{\mathrm{S}}\left(v_{j, k+1}^{\mathrm{S}}\right)^{2}-\rho_{j, k}^{\mathrm{N}}\left(v_{j, k}^{\mathrm{N}}\right)^{2}}{2}\right|, \\
& \Delta \beta_{j, k+\frac{1}{2}}^{(2)}=\left|\frac{\rho_{j, k+1}^{\mathrm{S}}\left(u_{j, k+1}^{\mathrm{S}}\right)^{2}-\rho_{j, k}^{\mathrm{N}}\left(u_{j, k}^{\mathrm{N}}\right)^{2}}{2}\right|, \quad \Delta \beta_{j, k+\frac{1}{2}}=\sqrt{\left(\Delta \beta_{j, k+\frac{1}{2}}^{(1)}\right)^{2}+\left(\Delta \beta_{j, k+\frac{1}{2}}^{(2)}\right)^{2}} .
\end{aligned}
$$

In (3.4) and (3.6), $\varepsilon$ is a small positive parameter designed to prevent division by zero.

Let us now turn back to the example with the initial setting outlined in Figure 1. It is easy to verify that after we replace the one-sided local speeds (3.2) with (3.3)-(3.5), all of the local speeds in the $x$-direction will reduce to $a_{j+\frac{1}{2}}^{ \pm} \equiv 0$ and the initial steady state will be exactly preserved at the discrete level. This simple example is designed to illustrate the main idea of the proposed switch. Its advantages will be further demonstrated in a variety of numerical examples in Section 4.

Remark 3.1. In order to minimize the risk of instability, we use the values $a_{j+\frac{1}{2}, k}^{ \pm}$and $b_{j, k+\frac{1}{2}}^{ \pm}$estimated in (3.2) (rather than their reduced versions given by (3.3)) in the computation of the time steps in (2.9) and (2.10).

\section{Numerical examples}

In this section, we demonstrate the performance of the proposed semi-discrete second-order central-upwind scheme with numerical dissipation switch on several numerical examples. In all of the experiments, we take the minmod parameter $\sigma=1.3$ and the switch threshold parameter $\varepsilon=10^{-12}$.

In the remaining part of the paper, we will refer to the proposed central-upwind scheme with the switch as "NEW scheme", while the original central-upwind scheme will be referred to as "OLD scheme".

\section{Example 1 - Moving contact wave}

In the first example, we numerically capture an isolated moving contact wave to compare the performance of the NEW and OLD schemes. To this end, we consider a 2-D contact wave on the computational domain $\Omega=[-0.2,0.2] \times[0,0.8]$ with free boundary conditions and the initial data given by

$$
(\rho(x, y, 0), u(x, y, 0), v(x, y, 0), p(x, y, 0))^{\top}= \begin{cases}(1.4,0,0.2,1)^{\top}, & (x, y) \in D \\ (1.0,0,0.2,1)^{\top}, & \text { otherwise. }\end{cases}
$$

Here, the domain $D$, outlined using the dashed line in Figure 4, consists of the points $(x, y) \in \Omega$ that satisfy the following conditions:

$$
\begin{aligned}
& \{-0.1<x<0.1,0<y<0.02\} \bigcup\{-0.02<x<0.02,0.02<y<0.1\} \bigcup \\
& \left\{(x+0.02)^{2}+(y-0.02)^{2}<0.08^{2}\right\} \bigcup\left\{(x-0.02)^{2}+(y-0.02)^{2}<0.08^{2}\right\} .
\end{aligned}
$$

We first proceed with the numerical simulations using the first-order scheme, which is obtained by replacing the point values given by (2.5) and (2.6) with the corresponding cell averages:

$$
\boldsymbol{U}_{j, k}^{\mathrm{E}}=\boldsymbol{U}_{j, k}^{\mathrm{W}}=\boldsymbol{U}_{j, k}^{\mathrm{N}}=\boldsymbol{U}_{j, k}^{\mathrm{S}}=\boldsymbol{U}_{j, k}^{\mathrm{NE}}=\boldsymbol{U}_{j, k}^{\mathrm{NW}}=\boldsymbol{U}_{j, k}^{\mathrm{SE}}=\boldsymbol{U}_{j, k}^{\mathrm{SW}}=\overline{\boldsymbol{U}}_{j, k}, \quad \forall(j, k) .
$$



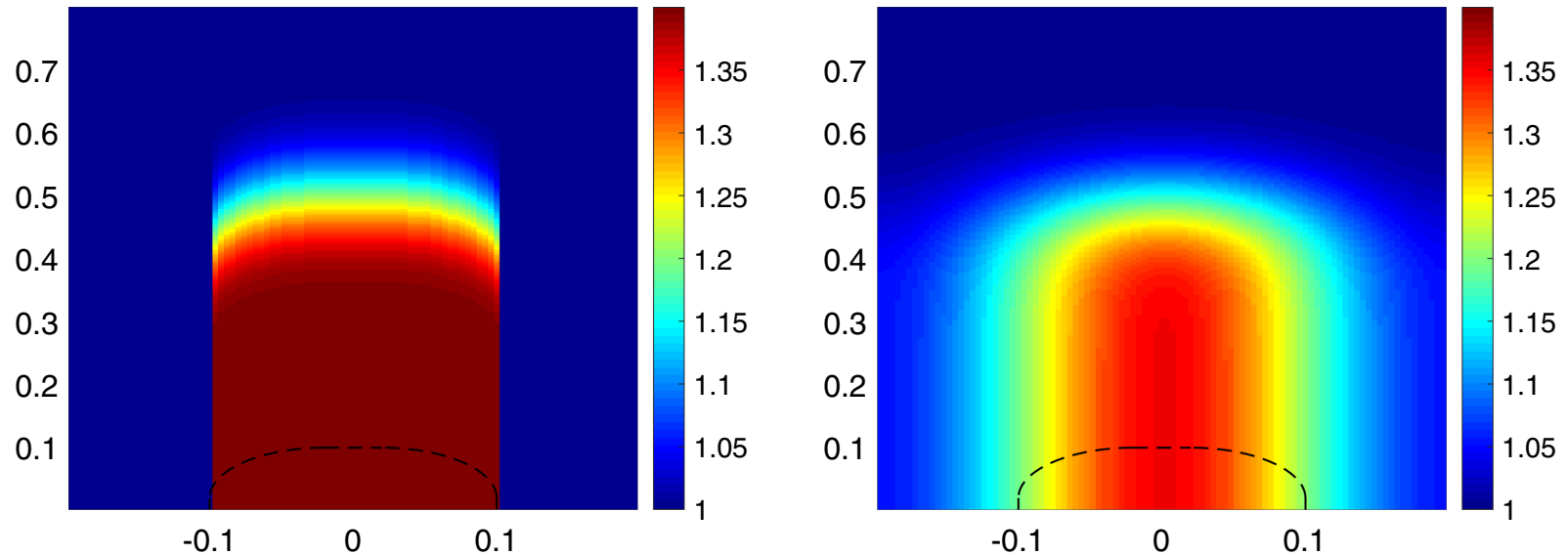

FiguRE 2. Example 1: Density $(\rho)$ computed by the first-order versions of the NEW (left) and OLD (right) schemes. The dashed line represents the initial contact line.
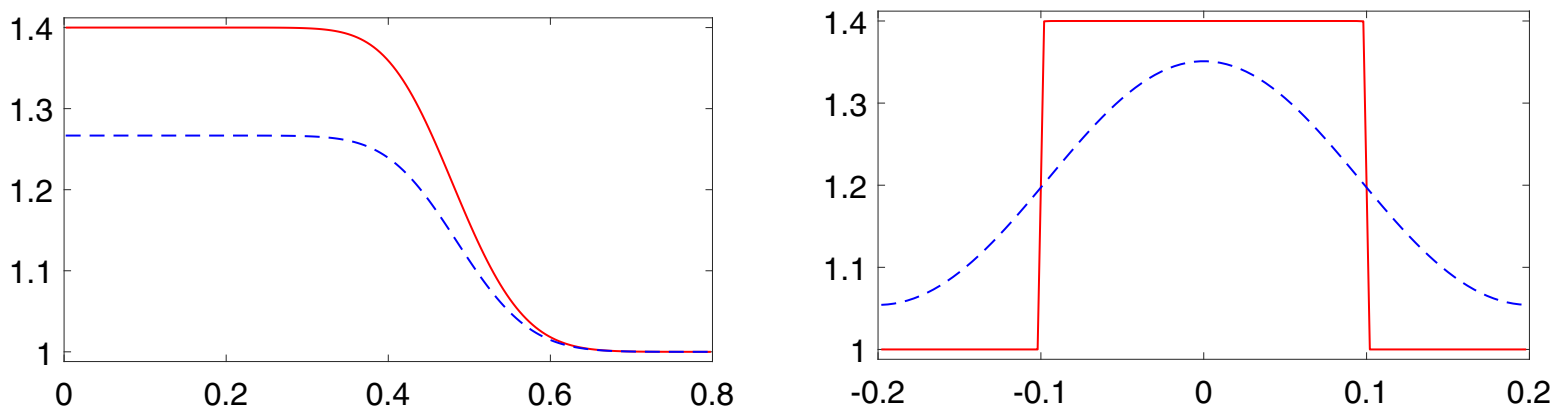

Figure 3. Example 1: 1-D slices along the lines $x=0.07$ (left) and $y=0.21$ (right) of the density $(\rho)$ computed by the first-order versions of the NEW (solid lines) and OLD (dashed lines) schemes.

The results (density) computed on the uniform grid with $\Delta x=\Delta y=2 / 500$ at the final time $t=2$ for both the NEW and OLD schemes are plotted in Figure 2. As one can clearly see, the proposed numerical dissipation switch helps to significantly reduce the numerical dissipation, especially at the contact waves (located at $x= \pm 0.1$ ), which are drastically smeared by the OLD scheme. This can be also clearly seen in Figure 3, where we plot the 1 -D slices of the computed solutions along the lines $x=0.07$ and $y=0.21$.

We then use the second-order schemes and plot the obtained results in Figure 4. As one can observe, the contact wave, especially its parts located along the lines $x= \pm 0.1$, captured much more accurately by the NEW scheme. This is confirmed in Figure 5, where we plot the 1-D slices of the computed solutions along the lines $x=0.07$ and $y=0.21$. Finally, we perform the mesh refinement study to check the difference in convergence of the NEW and OLD schemes. The obtained results are shown in Table 1, where one can see that the $L^{1}$-errors in the NEW scheme computations are about four times smaller than those obtained using the OLD scheme. At the same time, we notice that the convergence rates are about the same for both schemes, which is reasonable as the use of the proposed numerical dissipation switch is not expected to increase the order of convergence. 
TABLE 1. Example 1: $L^{1}$-errors in density and experimental convergence rates for both the NEW and OLD schemes.

\begin{tabular}{lllll}
\hline \hline$\Delta x=\Delta y$ & $\left\|\rho^{\text {exact }}-\rho^{\mathrm{NEW}}\right\|_{1}$ & Rate & $\left\|\rho^{\text {exact }}-\rho^{\mathrm{OLD}}\right\|_{1}$ & Rate \\
\hline $1 / 50$ & 0.0036 & - & 0.0222 & - \\
$1 / 100$ & 0.0023 & 0.66 & 0.0110 & 1.02 \\
$1 / 200$ & 0.0015 & 0.63 & 0.0063 & 0.80 \\
$1 / 400$ & 0.00081 & 0.85 & 0.0037 & 0.78 \\
\hline
\end{tabular}
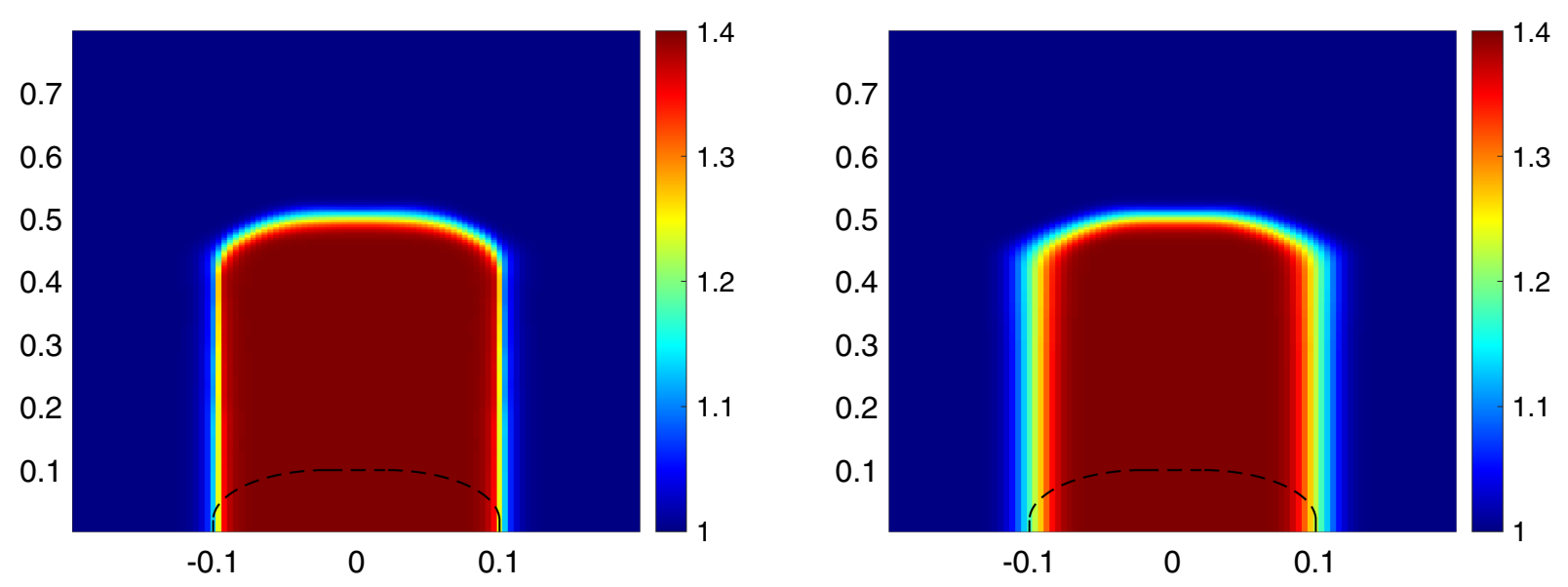

Figure 4. Example 1: Same as Figure 2 but for the second-order schemes.
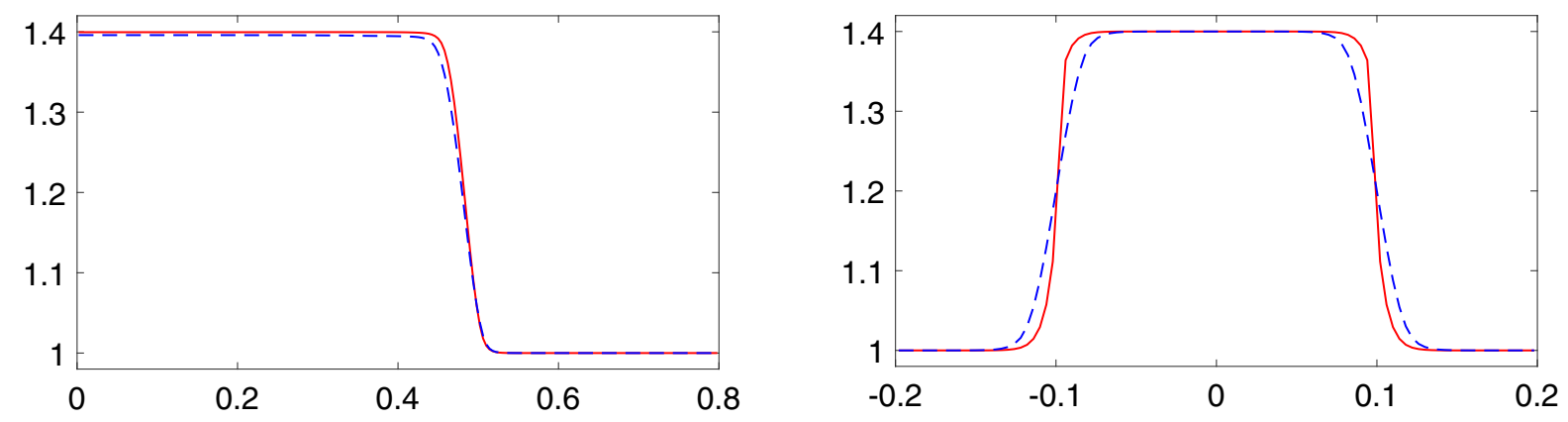

Figure 5. Example 1: Same as Figure 3 but for the second-order schemes.

\section{Example 2 - Accuracy test}

In the second example taken from $[3,4,39]$, we use the 2 -D inviscid isentropic vortex evolution problem with a diagonal flow to test the accuracy of both the NEW and OLD schemes.

We take the mean flow with $\rho=p=u=v \equiv 1$ and superimpose an isentropic vortex perturbation onto the velocity fields $(u, v)$ as well as the temperature $T=\frac{p}{\rho}$, while no perturbation is added to the entropy $S=\frac{p}{\rho^{\gamma}}$ :

$$
(\delta u, \delta v)=\frac{\eta}{2 \pi} e^{0.5\left(1-r^{2}\right)}(-\bar{y}, \bar{x}), \quad \delta T=-\frac{(\gamma-1) \eta^{2}}{8 \gamma \pi^{2}} e^{1-r^{2}}, \quad \delta S=0
$$


TABLE 2. Example 2: $L^{1}$-errors in density and experimental convergence rates for both the NEW and OLD schemes.

\begin{tabular}{lllll}
\hline \hline$\Delta x=\Delta y$ & $\left\|\rho^{\text {exact }}-\rho^{\mathrm{NEW}}\right\|_{1}$ & Rate & $\left\|\rho^{\text {exact }}-\rho^{\text {OLD }}\right\|_{1}$ & Rate \\
\hline $1 / 4$ & 0.3808 & - & 0.3996 & - \\
$1 / 8$ & 0.1221 & 1.65 & 0.1284 & 1.64 \\
$1 / 16$ & 0.0348 & 1.81 & 0.0357 & 1.85 \\
$1 / 32$ & 0.0097 & 1.85 & 0.0092 & 1.95 \\
\hline
\end{tabular}

where $(\bar{x}, \bar{y})=(x-5, y-5), r^{2}=\bar{x}^{2}+\bar{y}^{2}$, and $\eta$ represents the vortex strength. We take $\eta=5$ in our simulations. The computation domain is $[0,10] \times[0,10]$ and the periodic boundary conditions are imposed at all of the boundaries. The $L^{1}$-errors in density at $t=1$ are shown in Table 2 , which indicates that the both errors and convergence rates are about the same for both the NEW and OLD schemes. This demonstrates that the proposed numerical dissipation switch has almost no affect on computed smooth solutions.

\section{Example 3 - Two-dimensional Riemann problem}

In the third example, we consider the 2-D Riemann problems and test the proposed central-upwind scheme on all of the 19 configurations $^{1}$ from [24]; see also [30,47,48,55]. The obtained results demonstrate the robustness of the NEW scheme that generates the numerical solutions, which are not more oscillatory than the solutions computed by the OLD scheme or the central-upwind schemes from [24]. At the same time, the reduced numerical dissipation helps to improve the resolution of some parts of the computed solution. In order to illustrate this, we plot in Figure 6 the densities computed in Configurations 11, 13, 17 and 19. As one can see, there are some improvements achieved by the NEW scheme, especially in the resolution of contact waves.

We also perform a more detailed numerical study for one of the configurations - Configuration 3, considered in a slightly larger computational domain $[0,1.2] \times[0,1.2]$ discretized using about twice finer mesh with $1000 \times 1000$ uniform cells. The boundary conditions are the zero-order extrapolation and the initial data are given by

$$
(\rho, u, v, p)^{\top}= \begin{cases}(1.5,0,0,1.5)^{\top}, & x>1, y>1 \\ (0.5323,1.206,0,0.3)^{\top}, & x<1, y>1, \\ (0.1380,1.206,1.206,0.029)^{\top}, & x<1, y<1, \\ (0.5323,0,1.206,0.3)^{\top}, & x>1, y<1 .\end{cases}
$$

We compute the solutions until the final time $t=1$ and then plot the densities computed by the NEW and OLD schemes in Figure 7 (top row). As one can clearly see, both schemes maintain the diagonal symmetry of the guitar-like shape of the jet. However, we can clearly see that the NEW scheme, unlike the OLD one, captures a sideband instability of the jet, expected in the zones of strong along-jet velocity shear; see Figure 7 (bottom row), where we plot the velocity field.

It is instructive to take a look at the final time distributions of the switch parameters $\alpha_{j+\frac{1}{2}, k}$ and $\beta_{j, k+\frac{1}{2}}$, which are plotted in Figure 8. As one can observe, the parameters vary between 0 and 1 and their distributions clearly reflect the structure of the computed density shown in Figure 7 (left).

\footnotetext{
${ }^{1}$ These are genuinely different admissible configurations for polytropic gas, separated by the three types of 1-D centered waves, namely, rarefaction, shock and contact waves.
} 

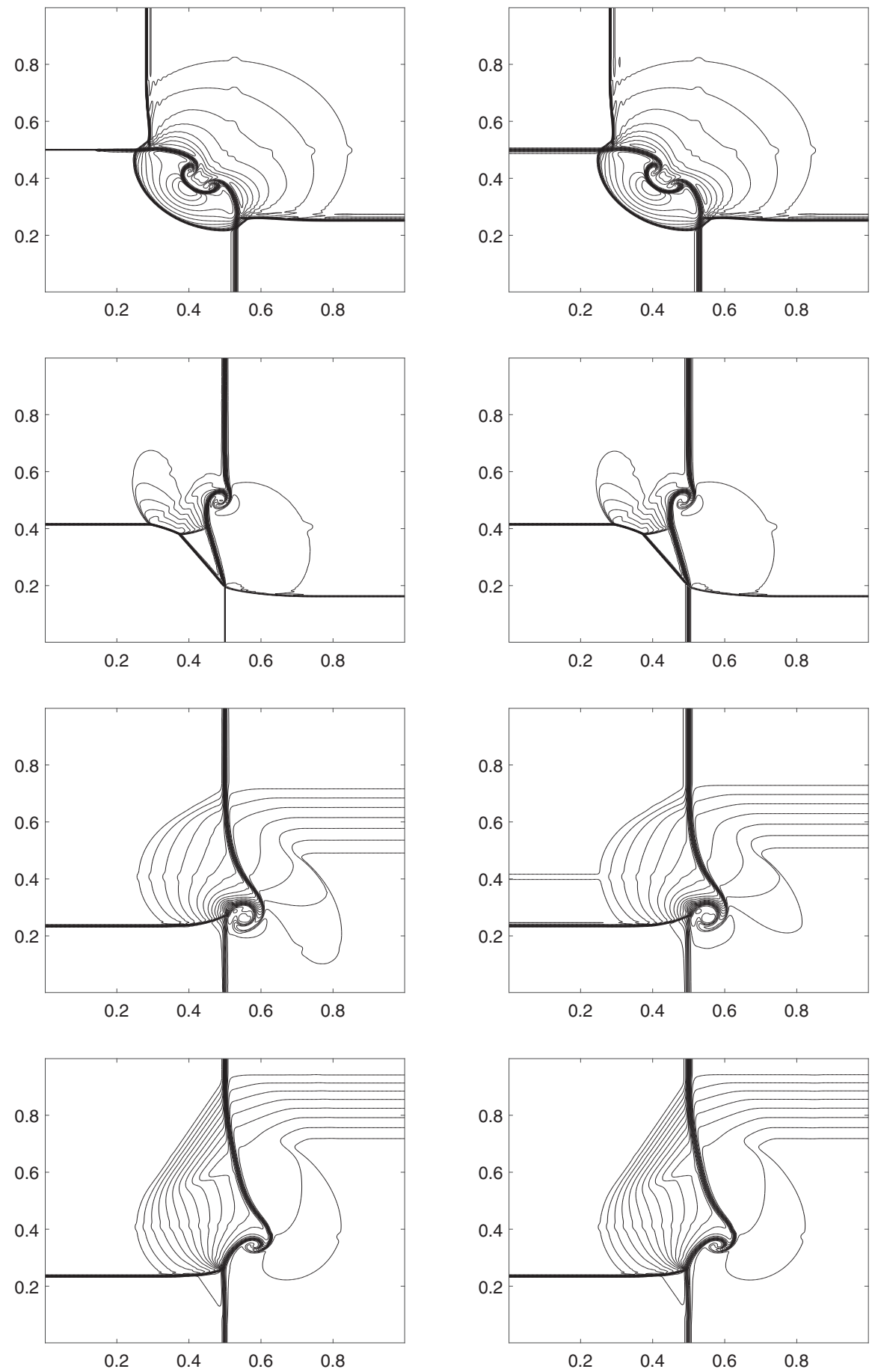

Figure 6. Example 3: Densities $(\rho)$ computed by the NEW (left column) and OLD (right column) schemes. From top to bottom: configurations 11, 13, 17 and 19. The initial settings, final times $(t=0.3)$ and mesh size $(400 \times 400$ cells in the $[0,1] \times[0,1]$ computational domain $)$ are identical to those used in [24]. 

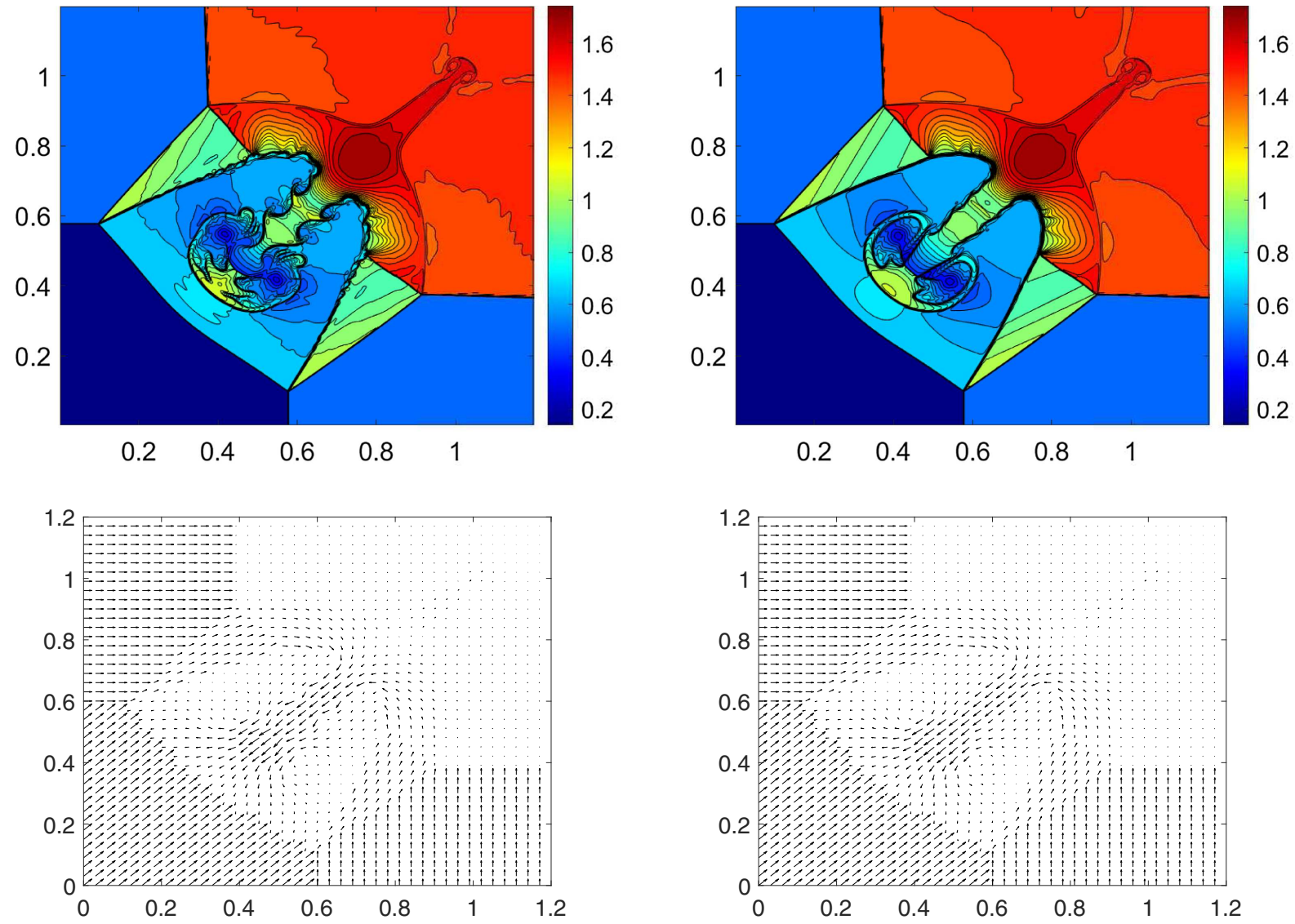

Figure 7. Example 3, Configuration 3: Density (top row) and velocity field (bottom row) computed by the NEW (left column) and OLD (right column) schemes.
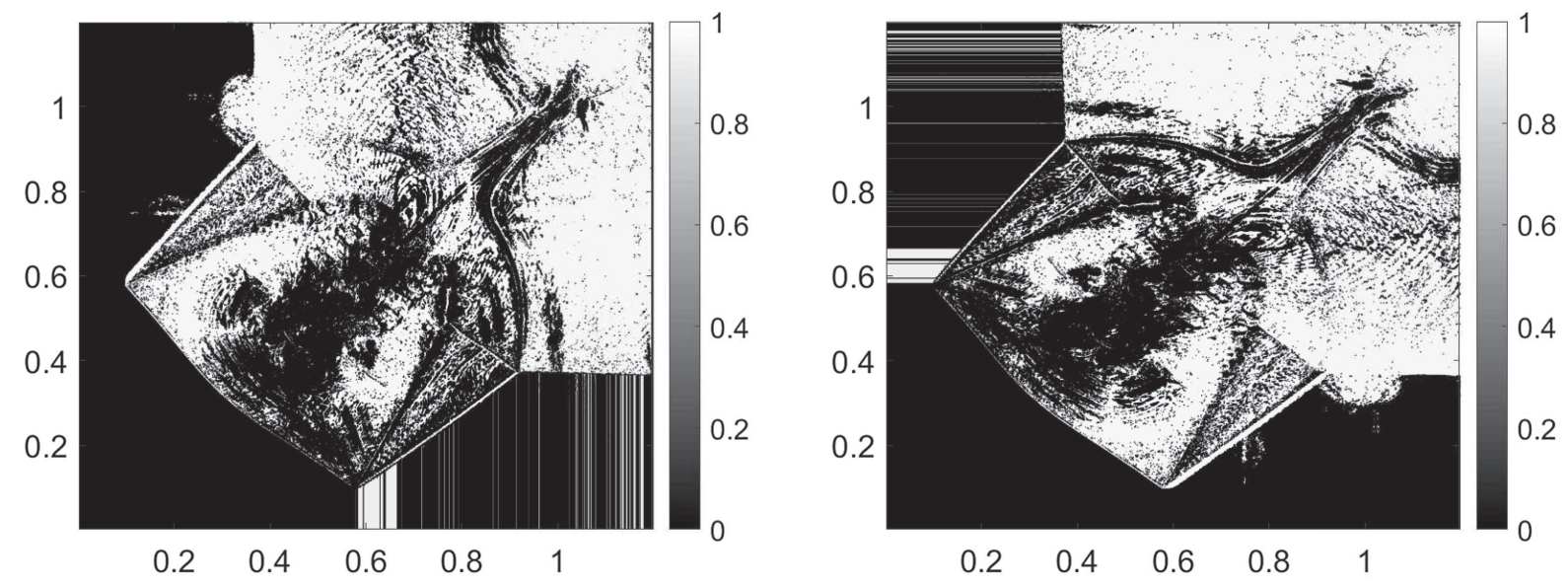

FiguRE 8. Example 3, Configuration 3: Switch parameters $\alpha_{j+\frac{1}{2}, k}$ (left) and $\beta_{j, k+\frac{1}{2}}$ (right). 

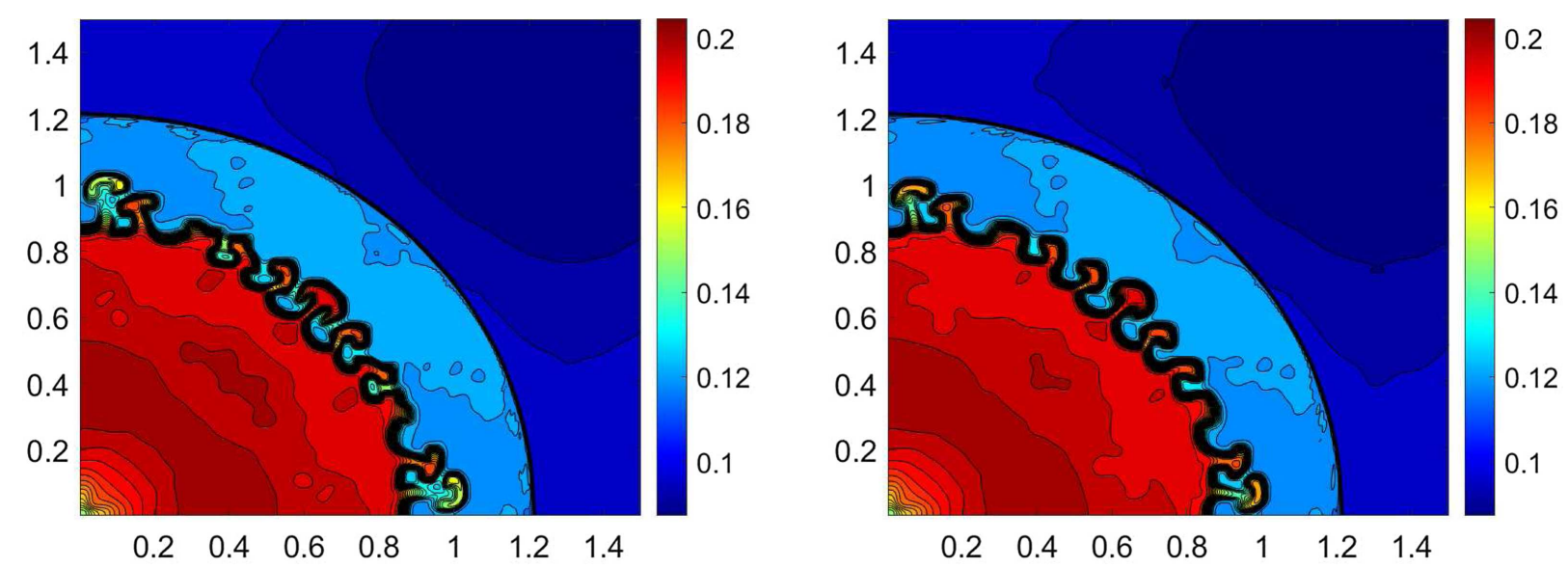

Figure 9. Example 4: Density $(\rho)$ computed by the NEW (left) and OLD (right) schemes.

\section{Example 4 - Explosion}

In the fourth example, which is taken from [38], the initial conditions are radially symmetric and given by

$$
(\rho(x, y, 0), u(x, y, 0), v(x, y, 0), p(x, y, 0))^{\top}= \begin{cases}(1.000,0,0,1.0)^{\top}, & x^{2}+y^{2}<0.16, \\ (0.125,0,0,0.1)^{\top}, & \text { otherwise. }\end{cases}
$$

The computational domain is $[0,1.5] \times[0,1.5]$. It is well-known (see, e.g., $[7,21,26,38]$ ) that the computed circular contact curve should develop instability by the time $t=3.2$ unless the numerical method contains an excessive amount of numerical dissipation. Therefore, this is a good test to compare the numerical dissipation present in the NEW and OLD schemes.

We solve the underlying problem using the uniform mesh with $\Delta x=\Delta y=3 / 800$ and set reflecting boundary conditions at $x=0$ and $y=0$ and free boundary conditions at $x=1.5$ and $y=1.5$. The $\rho$-component of the solutions obtained at time $t=3.2$ are plotted in Figure 9. As one can see there, both schemes produce mushroom-shaped structures along the contact surface. However, the instability structures produced by the NEW scheme are more developed than the ones produced by the OLD scheme. This demonstrates that the use of numerical dissipation switch helps to reduce the amount of numerical dissipation present in the central-upwind scheme. The final time distributions of the switch parameters $\alpha_{j+\frac{1}{2}, k}$ and $\beta_{j, k+\frac{1}{2}}$ are plotted in Figure 10. Note that a small reduction in the numerical dissipation does not lead to any instabilities in the neighborhood of the circular shock wave.

\section{Example 5 - Implosion}

In the fifth example, also taken from [38], we numerically solve the implosion problem in a square domain $[0,0.3] \times[0,0.3]$ and with solid wall boundary conditions. This test case is a challenging one because of the presence of non-grid-aligned shocks and emergence of a jet. The initial conditions are:

$$
(\rho(x, y, 0), u(x, y, 0), v(x, y, 0), p(x, y, 0))^{\top}= \begin{cases}(0.125,0,0,0.14)^{\top}, & |x|+|y|<0.15 \\ (1.000,0,0,1.00)^{\top}, & \text { otherwise. }\end{cases}
$$

As demonstrated in [38] (also see [7,26]), a jet of fluid is expected to emerge. However, the numerical diffusion may prevent the jet from appearing in the computed results or may artificially slow down its propagation speed. This makes the studied example to be an excellent test for comparing the amount of numerical dissipation present 

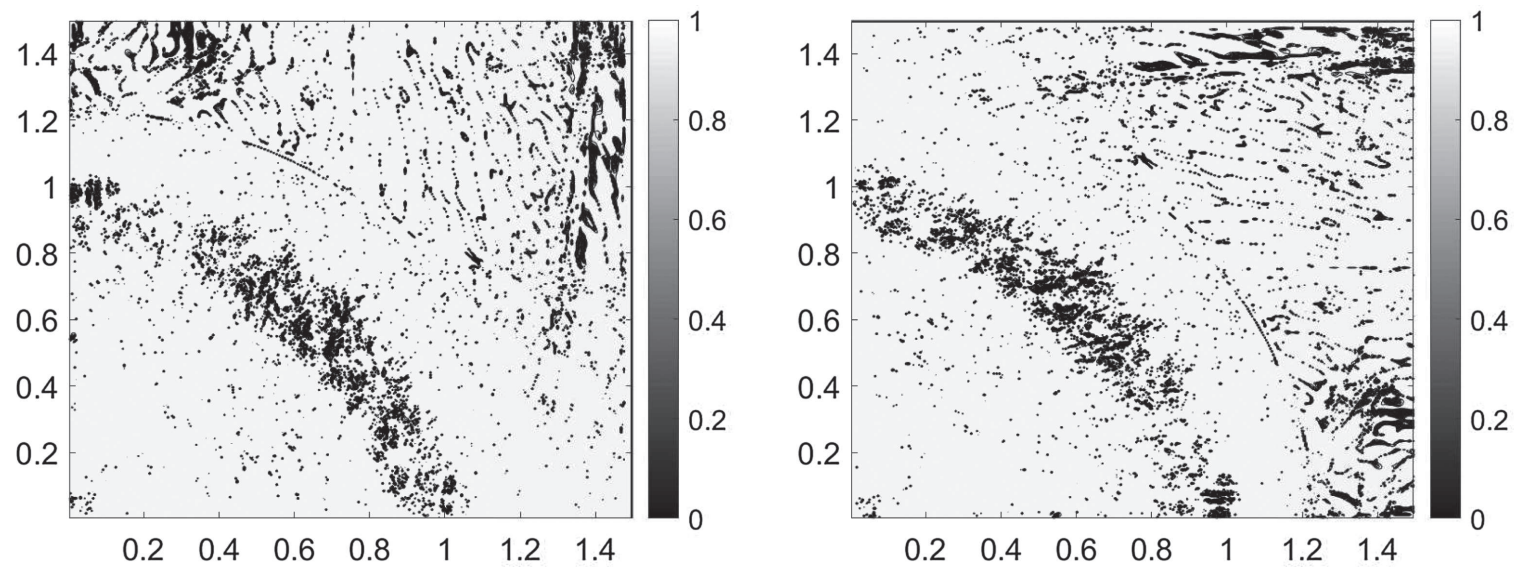

FiguRE 10. Example 4: Switch parameters $\alpha_{j+\frac{1}{2}, k}($ left $)$ and $\beta_{j, k+\frac{1}{2}}(r i g h t)$.
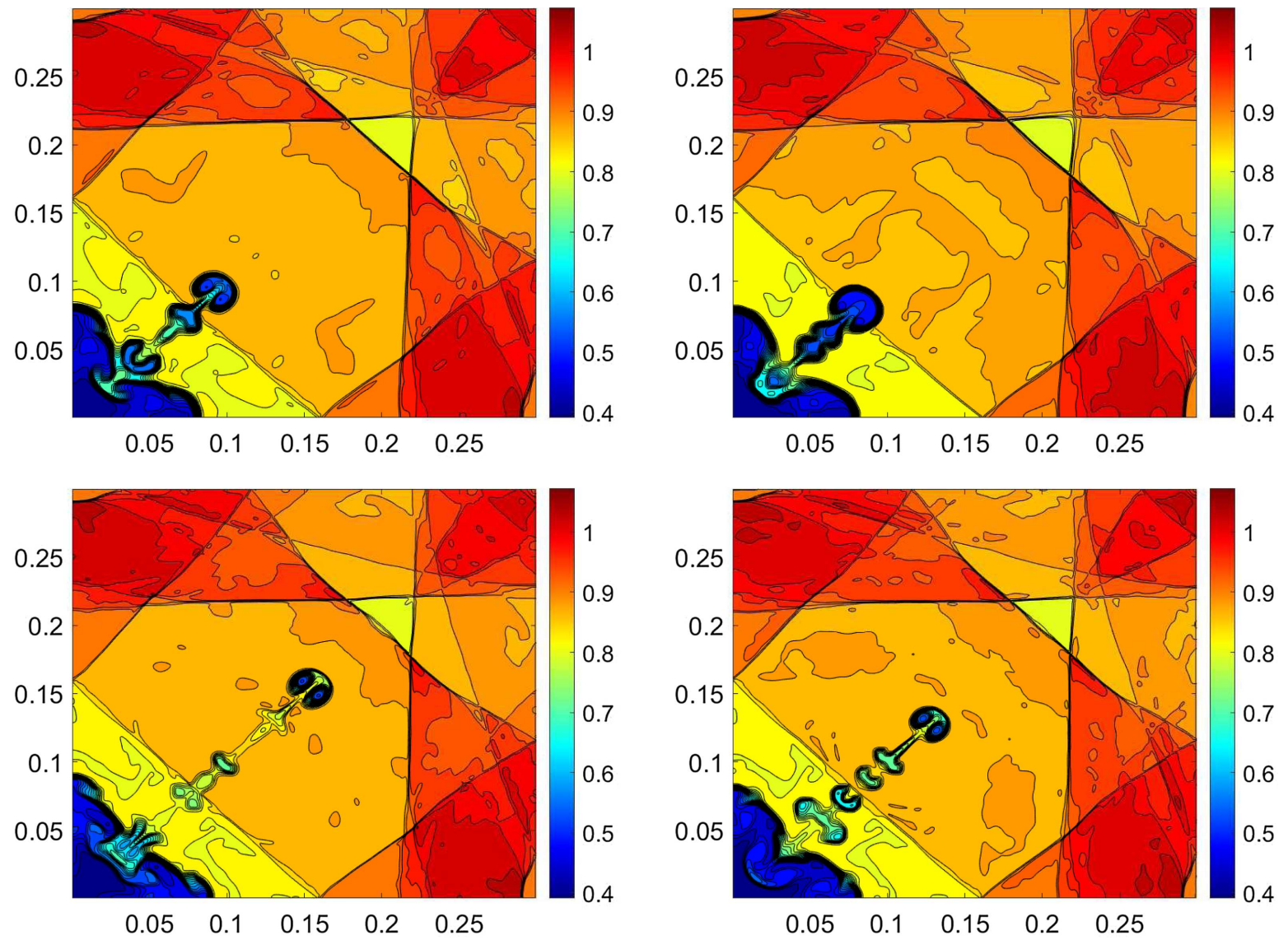

Figure 11. Example 5: Density $(\rho)$ computed by the NEW (left column) and OLD (right column) schemes using a coarser (with $\Delta x=\Delta y=3 / 6000$; upper row) and finer (with $\Delta x=$ $\Delta y=3 / 8000$; lower row) meshes. 

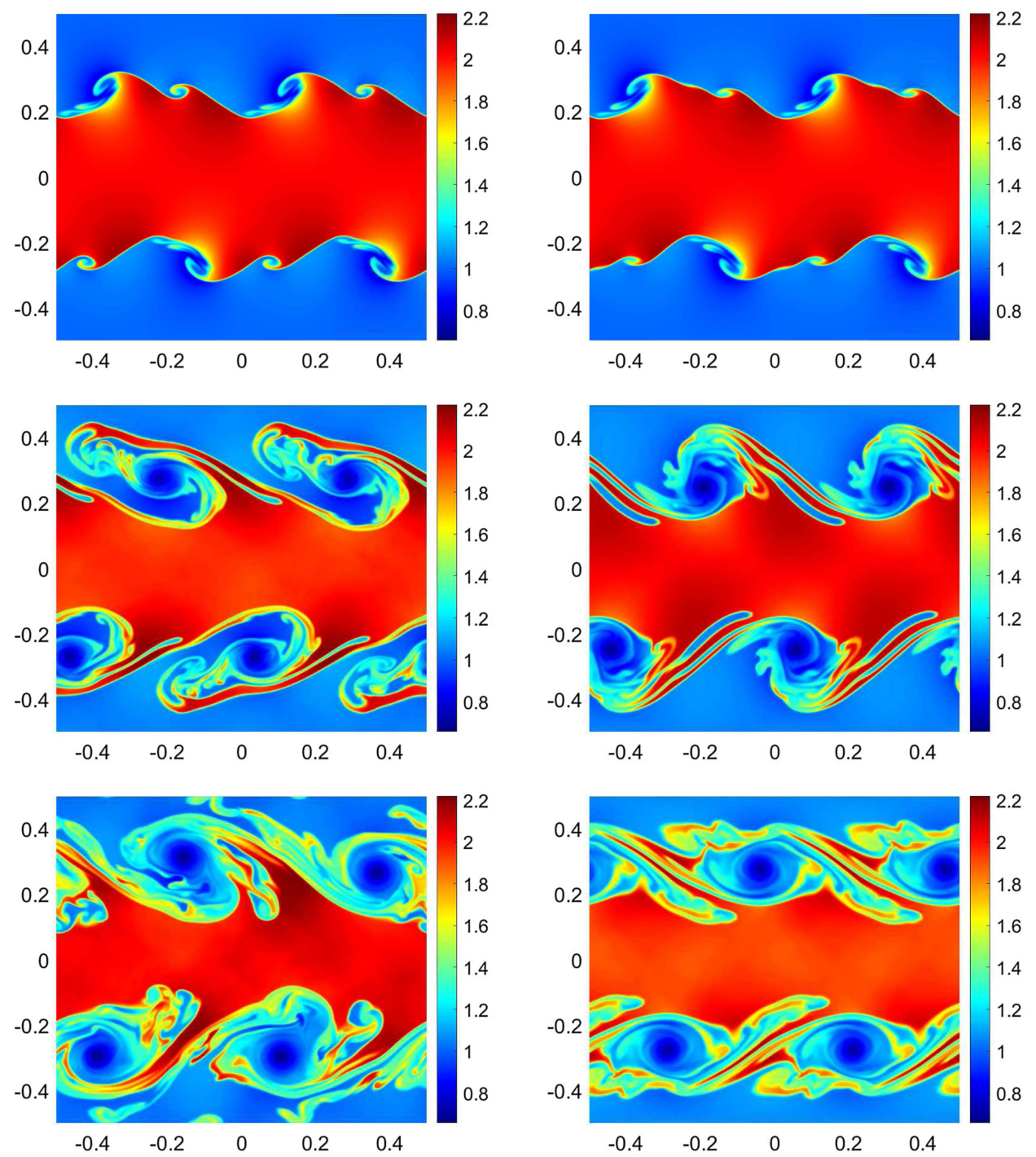

Figure 12. Example 6: Density $(\rho)$ computed by the NEW (left column) and OLD (right column) schemes at times $t=1$ (top row), 2.5 (middle row) and 4 (bottom row).

in the NEW and OLD schemes. We first compute the solution on the uniform grid with $\Delta x=\Delta y=3 / 6000$ until the final time $t=2.5$. As one can see in the upper row of Figure 11, the jet produced by the NEW scheme develops faster. If we refine the mesh by taking $\Delta x=\Delta y=3 / 8000$, the amount of numerical dissipation reduces in both the schemes. As a result, the computed jets propagate further towards the center of the computational domain, but the NEW scheme is still clearly less dissipative than the OLD one; see the lower row of Figure 11, where one can observe that the jet produced by the NEW scheme propagates substantially further.

\section{Example 6 - Kelvin-Helmholtz (KH) instability}

In the sixth example, we numerically study the $\mathrm{KH}$ instability, which is taken from [43], and also studied in [8]. The KH instability occurs when a shearing flow is slightly perturbed and it will generate internal waves, 

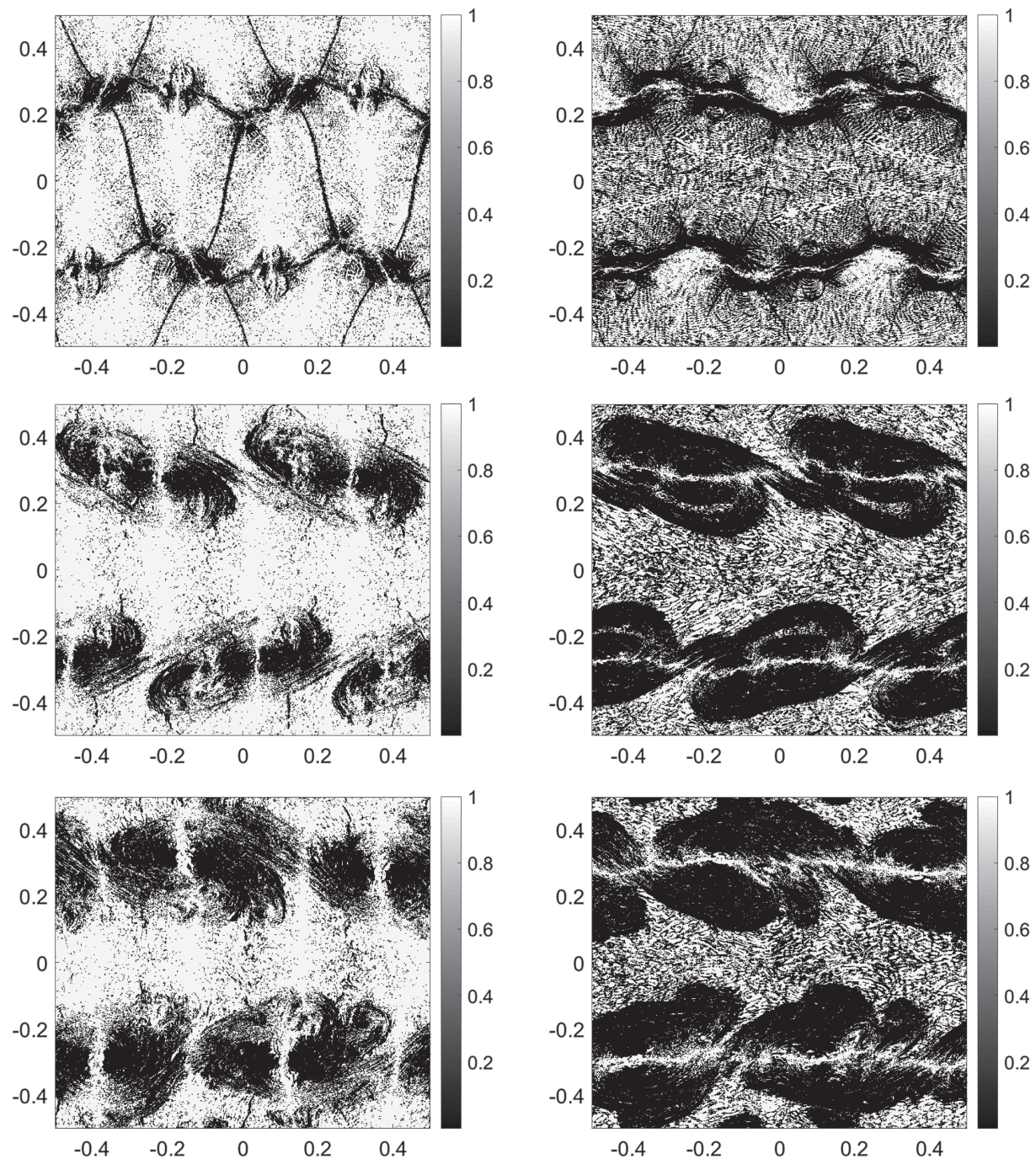

FiguRE 13. Example 6: Switch parameters $\alpha_{j+\frac{1}{2}, k}$ (left column) and $\beta_{j, k+\frac{1}{2}}$ (right column) at times $t=1$ (top row), 2.5 (middle row) and 4 (bottom row).

which are growing, breaking and forming vortices. This is the reason why we can see some beautiful breaking wave-like cloud patterns or billow clouds in the sky. Here, we study the KH instability to demonstrate that the NEW scheme has much better ability to capture the formation of a wave pattern then the OLD one. To this end, we consider the following initial conditions in [43]:

$$
(\rho(x, y, 0), u(x, y, 0))= \begin{cases}\left(1,-0.5+0.5 e^{(y+0.25) / L}\right), & y \in[-0.5,-0.25), \\ \left(2,0.5-0.5 e^{(-y-0.25) / L}\right), & y \in[-0.25,0), \\ \left(2,0.5-0.5 e^{(y-0.25) / L}\right), & y \in[0,0.25), \\ \left(1,-0.5+0.5 e^{(0.25-y) / L}\right), & y \in[0.25,0.5],\end{cases}
$$



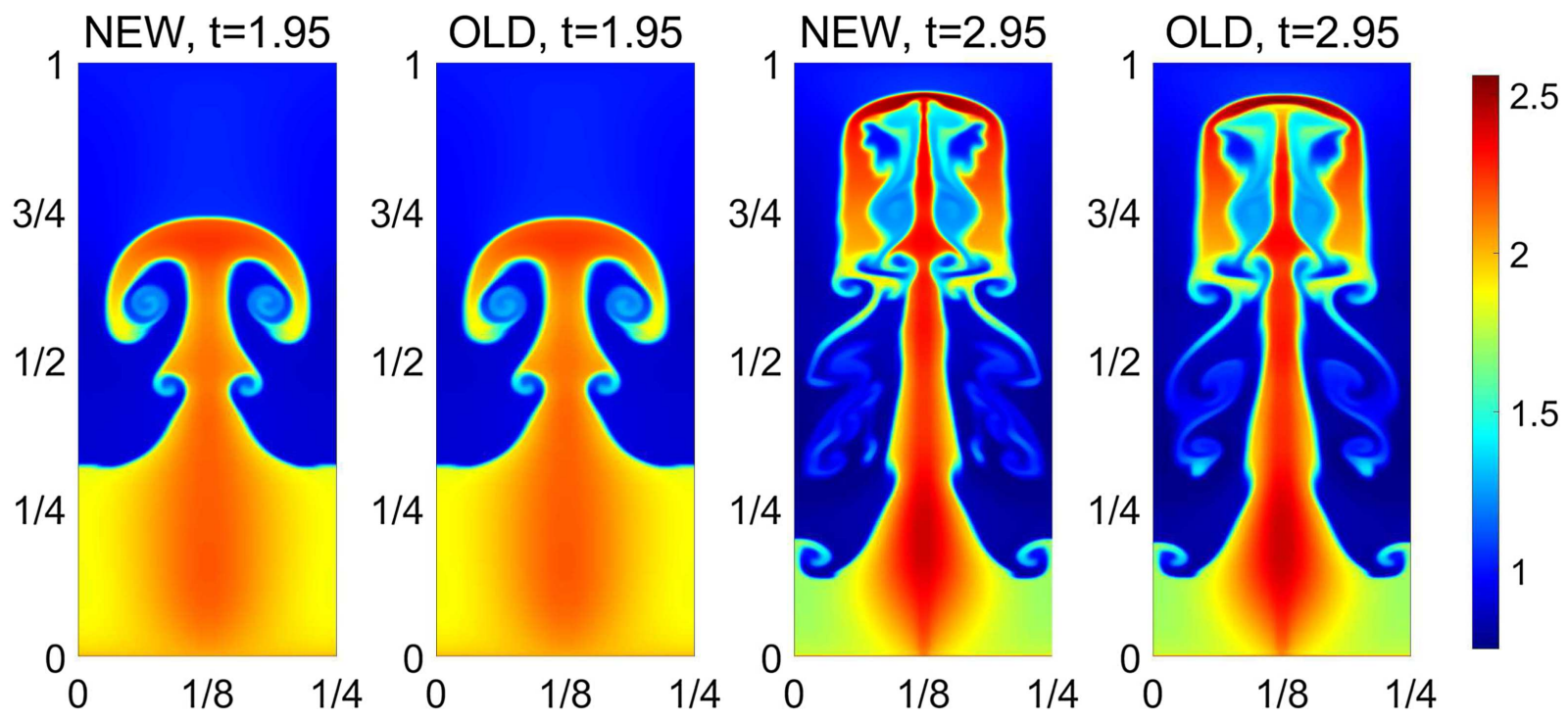

Figure 14. Example 7: Density $(\rho)$ computed by the NEW and OLD schemes.
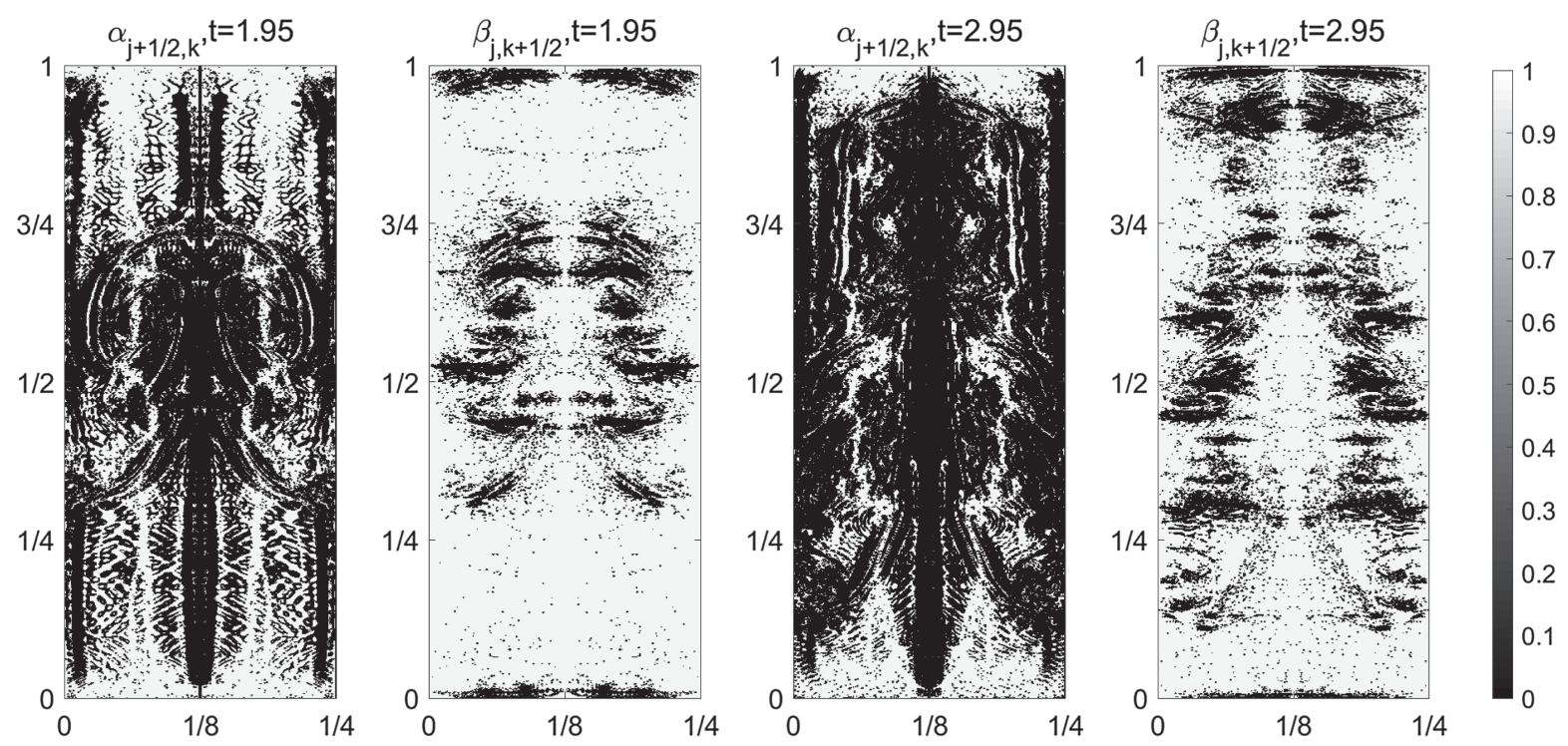

FIGURE 15. Example 7: Switch parameters $\alpha_{j+\frac{1}{2}, k}$ and $\beta_{j, k+\frac{1}{2}}$.

where $L$ is a smoothing parameter and we take $L=0.00625$ corresponding to a thin shear interface in the simulation below. Besides, the initial pressure $p(x, y, 0)=1.5$ and the vertical velocity is

$$
v(x, y, 0)=0.01 \sin (4 \pi x) .
$$

The computation domain is $[-0.5,0.5] \times[-0.5,0.5]$. We use a uniform grid with $\Delta x=\Delta y=1 / 1024$ and the periodic boundary conditions to compute the solutions until time $t=4$. The slices of density $\rho$ at times $t=1$, 2.5 and 4 are plotted in Figure 12. We can clearly see that, at $t=1$, the NEW scheme capture slightly more 
vortices than the OLD one, especially the small wavy bumps at the position around $(-0.15,0.26),(0.35,0.26)$, $(-0.42,-0.28)$ and $(0.07,-0.28)$. From these four positions, we observe that the NEW scheme produce more clear swirl structures. At later time $t=2.5$, the difference between the proposed NEW scheme and the OLD scheme is apparently obvious. As one can see, the NEW scheme resolves secondary instabilities of KH billows and resolves more details in the wave-breaking pattern, among other things. However, these are much more fuzzy when computed by the OLD scheme. Furthermore, one can observe that the vortices produced by the NEW scheme move faster than the those captured by the OLD scheme. At the final time $t=4$, we can first see that the results of the NEW scheme are much less diffusive, as the red color is much darker which corresponding lowdissipation results. Second, we find that the NEW scheme produces more isolated and complicated vorticities indicating that this scheme can capture the KH instability much better than the OLD one. The time evolution of the switch parameters $\alpha_{j+\frac{1}{2}, k}$ and $\beta_{j, k+\frac{1}{2}}$ is shown in Figure 13, where one can see that the parameters near the rapidly changing parts of the solution are less than 1 , which helps to reduce the numerical diffusion there.

\section{Example 7 - Raleigh-Taylor (RT) instability}

In the seventh example, we numerically investigate the RT instability, which is a common test for accuracy studies of numerical schemes and contains both discontinuities and complex flow structure. Instead of (3.1), we include a source terms acting downward in the $y$-direction. In fact, we solve the following 2-D system:

$$
\begin{aligned}
\rho_{t}+(\rho u)_{x}+(\rho v)_{y} & =0, \\
(\rho u)_{t}+\left(\rho u^{2}+p\right)_{x}+(\rho u v)_{y} & =0, \\
(\rho v)_{t}+(\rho u v)_{x}+\left(\rho v^{2}+p\right)_{y} & =\rho, \\
E_{t}+[u(E+p)]_{x}+[v(E+p)]_{y} & =\rho v .
\end{aligned}
$$

We follow the setting in [49] and consider the following initial conditions:

$$
(\rho, u, v, p)(x, y, 0)= \begin{cases}(2,0,-0.025 c \cos (8 \pi x), 2 y+1), & y<0.5 \\ (1,0,-0.025 c \cos (8 \pi x), y+1.5), & \text { otherwise }\end{cases}
$$

where $c=\sqrt{\frac{\gamma p}{\rho}}$ is the sound speed. We should remark that in this example, we take the ratio of specific heats $\gamma=\frac{5}{3}$. The computation is prescribed in the domain $[0,1 / 4] \times[0,1]$ and reflective boundary conditions on left and right border lines are imposed. In addition, top and bottom boundary states are fixed to $(1,0,0,2.5)$ and $(2,0,0,1)$, respectively.

This test is a useful one to verify numerical methods, as the resolution and richness of vortical structures of this test case can be utilized as a measure of the numerical dissipation introduced by the methods. In Figure 14, we use $\Delta x=\Delta y=1 / 1024$ to compare the solutions computed by the above two schemes at the times $t=1.95$ and 2.95, respectively. As one can see, at earlier time $t=1.95$, there are no obvious differences between two schemes. However, at later time $t=2.95$, the difference is so obvious that we can clearly see the NEW scheme gives a much better resolution in complicated solution structures than the OLD one with same mesh size. It demonstrates that the more dissipative OLD scheme suppresses the Rayleigh-Taylor instability and artificially stabilizes the contact surface, while the NEW scheme can obtain the higher resolution for the complicated flow structure. Finally, the time evolution of the switch parameters $\alpha_{j+\frac{1}{2}, k}$ and $\beta_{j, k+\frac{1}{2}}$, presented in Figure 15, clearly demonstrates that these parameters are self-adapted to the developing structures of the computed solution.

Remark 4.1. In order to numerically preserve the symmetry property of the computational results, in our experiments we rewrite the initial velocity in the $y$-direction in the following symmetric form:

$$
v(x, y, 0)= \begin{cases}-0.025 c \cos (8 \pi x), & \text { if } x<0.125 \\ -0.025 c \cos (8 \pi(0.25-x)), & \text { otherwise }\end{cases}
$$



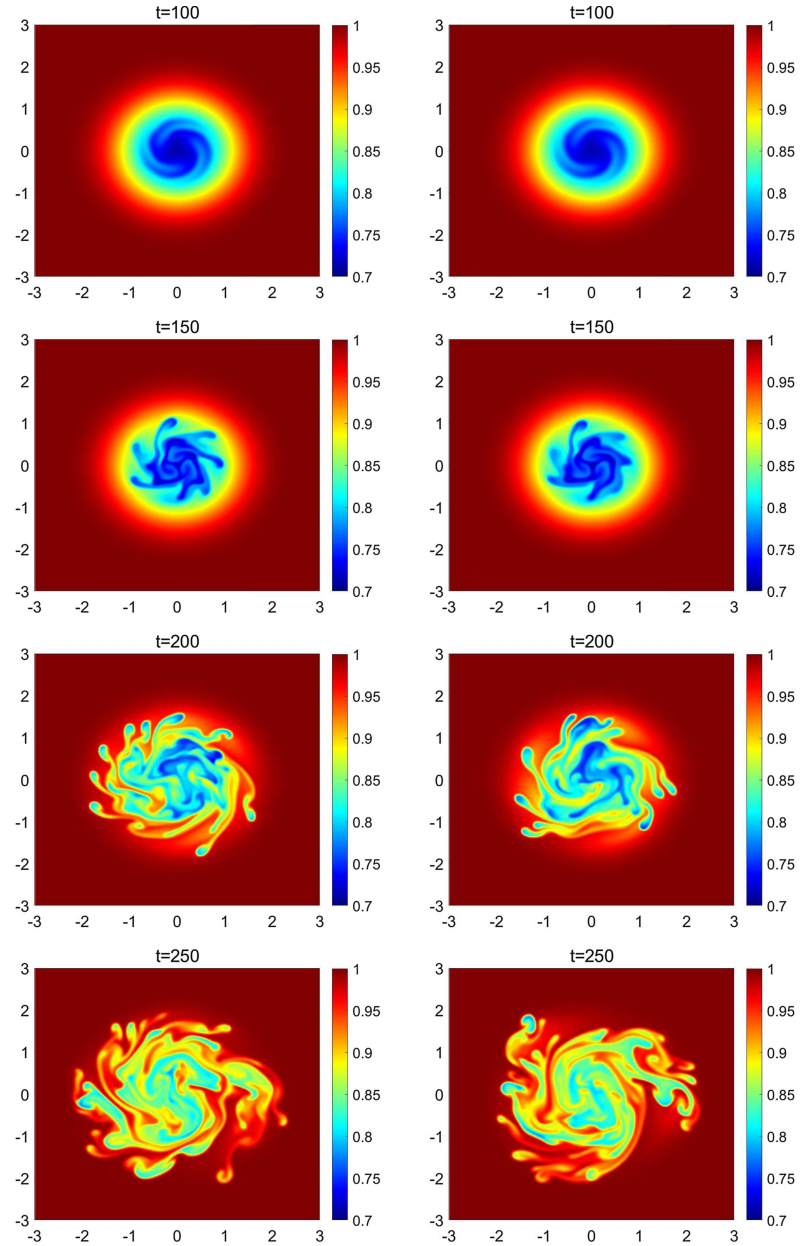

FiguRE 16. Example 8: Time snapshots of buoyancy $(\theta)$ computed by the NEW scheme (left) and OLD scheme (right) using $\Delta x=\Delta y=1 / 100$ and Neumann boundary conditions.

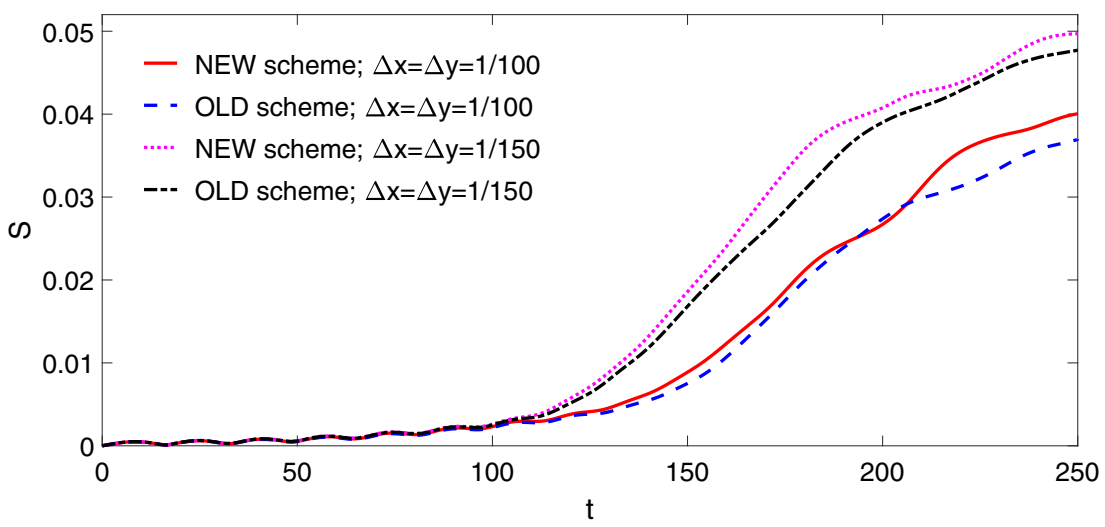

Figure 17. Example 8: The standard deviation $\mathcal{S}(t)$ defined in $(4.3)$ and computed by the NEW and OLD schemes using two different uniform meshes. 


\section{Example 8 - Vortex instability in thermal rotating shallow water equations}

In the final numerical example, we consider the 2 -D thermal rotating shallow water equations (see $[27,28]$ ):

$$
\begin{aligned}
h_{t}+(h u)_{x}+(h v)_{y} & =0, \\
(h u)_{t}+\left(h u^{2}+\frac{\theta}{2} h^{2}\right)_{x}+(h u v)_{y} & =f h v+h \theta Z_{x}, \\
(h v)_{t}+(h u v)_{x}+\left(h v^{2}+\frac{\theta}{2} h^{2}\right)_{y} & =-f h u-h \theta Z_{y}, \\
(h \theta)_{t}+(h u \theta)_{x}+(h v \theta)_{y} & =0,
\end{aligned}
$$

which was repeatedly rediscovered and used in the literature both in the atmospheric and oceanic context; see, e.g., $[6,29,41,44,46,53,54]$. In (4.1), $h$ denotes the thickness of the fluid layer, $Z$ represents the bottom topography, $u$ and $v$ stand for the zonal and meridional velocities, respectively, $\theta$ is the relative potential temperature, and $f(y)$ is the Coriolis parameter and taken to be $f(y) \equiv 1$ in this example.

We consider the vortex instability test, which was introduced in [13] as a benchmark crash test for numerical schemes; see $[13,27]$ for more details. The initial conditions are given by

$$
h(r, 0) \equiv 1, \quad \theta(r, 0)=1-\frac{1}{5} \int_{r}^{\infty}\left(1+\frac{V\left(r^{\prime}\right)}{10 r^{\prime}}\right) \mathrm{d} r^{\prime}, \quad u(r, 0)=-\frac{y}{r} V(r), \quad v(r, 0)=\frac{x}{r} V(r),
$$

where $r=\sqrt{x^{2}+y^{2}}$, and $V(r)=r \exp \left(-\left(r^{3}-1\right) / 3\right)$ is the nondimensional velocity. We note that the unperturbed vortex (4.2) does not preserve its initial structure for very long time and thus one cannot clearly see the appearance of instability. We therefore follow the approach in $[13,27]$ and superimpose the purely thermal vortex with an unstable mode with azimuthal wavenumber $n=3$ and a small amplitude of the order of one percent of the background vortex field. In Figure 16, we plot the relative potential temperature $\theta$ computed by the NEW and OLD schemes in the computational domain $[-3,3] \times[-3,3]$ at times $t=100,150,200$ and 250 . We notice that, at an earlier time $t=100$, one cannot see any visible difference between the results obtained by the both schemes. However, at later times $t=150,200$ and 250, one can clearly see that the NEW scheme produces more complicated branches than the OLD one, and the difference between the solutions computed by the two studied schemes becomes more and more obvious as time increases. Furthermore, the convective instabilities develop much faster and become more evident in the NEW scheme results.

On the other hand, in order to see the advantages of the NEW scheme in a more quantitative way, we compute the standard deviation of the potential temperature $\theta$ from its initial value:

$$
\mathcal{S}(t)=\sqrt{\frac{1}{N_{x} N_{y}} \sum_{j=1}^{N_{x}} \sum_{k=1}^{N_{y}}\left[\theta_{j, k}(t)-\theta_{j, k}(0)\right]^{2}},
$$

where $N_{x} \times N_{y}$ is a number of computational cells. We expect that the less-dissipative NEW scheme will produce a larger standard deviation as the less-dissipative solution is expected to develop more complex structures. In Figure 17, we plot $\mathcal{S}(t)$ computed by the NEW and OLD schemes using coarser and finer uniform meshes with $\Delta x=\Delta y=1 / 100$ and $\Delta x=\Delta y=1 / 150$, respectively. As one can clearly see, higher resolution solutions indeed have larger standard deviation. We also observe that the solutions computed by the NEW scheme have larger standard deviation than the ones computed by the OLD scheme on the same mesh. This means that the OLD scheme underestimates the energy of the small-scale turbulent fluctuations produced by developing convective instabilities, which is an important information in view of the applications, for instance, to the oceanic mixed layer.

Remark 4.2. A detailed information about the thermal rotating shallow water equations, flux globalization based central-upwind scheme for them, and the corresponding numerical dissipation switch can be found in $[27,28]$. In Example 8, we only illustrate the effect of the numerical dissipation switch. 
Acknowledgements. The work of A. Kurganov was supported in part by NSFC grant 11771201 and by the fund of the Guangdong Provincial Key Laboratory of Computational Science and Material Design (No. 2019B030301001). The work of A. Kurganov and V. Zeitlin was supported in part by the French National Program LEFE-MANU.

\section{REFERENCES}

[1] P. Arminjon, M.-C. Viallon and A. Madrane, A finite volume extension of the Lax-Friedrichs and Nessyahu-Tadmor schemes for conservation laws on unstructured grids. Int. J. Comput. Fluid Dyn. 9 (1997) 1-22.

[2] D.S. Balsara and B. Nkonga, Multidimensional Riemann problem with self-similar internal structure - part III - a multidimensional analogue of the HLLI Riemann solver for conservative hyperbolic systems. J. Comput. Phys. 346 (2017) $25-48$.

[3] J. Cheng and C.-W. Shu, A high order ENO conservative Lagrangian type scheme for the compressible Euler equations. J. Comput. Phys. 227 (2007) 1567-1596.

[4] J. Cheng and C.-W. Shu, A third order conservative Lagrangian type scheme on curvilinear meshes for the compressible Euler equations. Commun. Comput. Phys. 4 (2008) 1008-1024.

[5] A. Chertock, S. Cui, A. Kurganov, Ş. N. Özcan and E. Tadmor, Well-balanced schemes for the Euler equations with gravitation: Conservative formulation using global fluxes. J. Comput. Phys. 358 (2018) 36-52.

[6] D.P. Dempsey and R. Rotunno, Topographic generation of mesoscale vortices in mixed-layer models. J. Atmos. Sci. 45 (1988) 2961-2978.

[7] J. Dewar, A. Kurganov and M. Leopold, Pressure-based adaption indicator for compressible Euler equations. Numer. Methods Part. Differ. Equ. 31 (2015) 1844-1874.

[8] U.S. Fjordholm, S. Mishra and E. Tadmor, On the computation of measure-valued solutions. Acta Numer. 25 (2016) 567-679.

[9] E. Godlewski and P.-A. Raviart, Numerical approximation of hyperbolic systems of conservation laws. In: Vol. 118 of Applied Mathematical Sciences. Springer-Verlag, New York (1996).

[10] S.K. Godunov, A difference method for numerical calculation of discontinuous solutions of the equations of hydrodynamics. Mat. Sb. (N.S.) 47 (1959) 271-306.

[11] S. Gottlieb, C.-W. Shu and E. Tadmor, Strong stability-preserving high-order time discretization methods. SIAM Rev. 43 (2001) 89-112.

[12] S. Gottlieb, D. Ketcheson and C.-W. Shu, Strong Stability Preserving Runge-Kutta and Multistep Time Discretizations. World Scientific Publishing Co. Pte. Ltd., Hackensack, NJ (2011).

[13] E. Gouzien, N. Lahaye, V. Zeitlin and T. Dubos, Thermal instability in rotating shallow water with horizontal temperature/density gradients. Phys. Fluids 29 (2017) 101702.

[14] J.-L. Guermond and B. Popov, Fast estimation from above of the maximum wave speed in the Riemann problem for the Euler equations. J. Comput. Phys. 321 (2016) 908-926.

[15] A. Harten, P. Lax and B. van Leer, On upstream differencing and Godunov-type schemes for hyperbolic conservation laws. SIAM Rev. 25 (1983) 35-61.

[16] J.S. Hesthaven, Numerical methods for conservation laws. From analysis to algorithms. In: Vol. 18 of Computational Science 86 Engineering. Society for Industrial and Applied Mathematics (SIAM). Philadelphia, PA (2018).

[17] G.-S. Jiang and E. Tadmor, Nonoscillatory central schemes for multidimensional hyperbolic conservation laws. SIAM J. Sci. Comput. 19 (1998) 1892-1917.

[18] D. Kröner, Numerical Schemes for Conservation Laws. Wiley-Teubner Series Advances in Numerical Mathematics. John Wiley \& Sons Ltd., Chichester (1997).

[19] A. Kurganov, Central schemes: a powerful black-box solver for nonlinear hyperbolic PDEs. In: Vol. 17 of Handbook of Numerical Methods for Hyperbolic Problems, Handb. Numer. Anal. Elsevier/North-Holland, Amsterdam (2016) 525-548.

[20] A. Kurganov, Finite-volume schemes for shallow-water equations. Acta Numer. 27 (2018) 289-351.

[21] A. Kurganov and C.-T. Lin, On the reduction of numerical dissipation in central-upwind schemes. Commun. Comput. Phys. 2 (2007) 141-163.

[22] A. Kurganov and G. Petrova, A third-order semi-discrete genuinely multidimensional central scheme for hyperbolic conservation laws and related problems. Numer. Math. 88 (2001) 683-729.

[23] A. Kurganov and E. Tadmor, New high-resolution central schemes for nonlinear conservation laws and convection-diffusion equations. J. Comput. Phys. 160 (2000) 241-282.

[24] A. Kurganov and E. Tadmor, Solution of two-dimensional Riemann problems for gas dynamics without Riemann problem solvers. Numer. Methods Part. Differ. Equ. 18 (2002) 584-608.

[25] A. Kurganov, S. Noelle and G. Petrova, Semidiscrete central-upwind schemes for hyperbolic conservation laws and HamiltonJacobi equations. SIAM J. Sci. Comput. 23 (2001) 707-740.

[26] A. Kurganov, M. Prugger and T. Wu, Second-order fully discrete central-upwind scheme for two-dimensional hyperbolic systems of conservation laws. SIAM J. Sci. Comput. 39 (2017) A947-A965.

[27] A. Kurganov, Y. Liu and V. Zeitlin, Thermal vs isothermal rotating shallow water equations: comparison of dynamical processes in two models by simulations with a novel well-balanced central-upwind scheme. To appear in: Geophys. Astro. Fluid Dyn. (2020) DOI: 10.1080/03091929.2020.1774876.

[28] A. Kurganov, Y. Liu and V. Zeitlin, A well-balanced central-upwind scheme for the thermal rotating shallow water equations. J. Comput. Phys. 411 (2020) 109414. 
[29] R.L. Lavoie, A mesoscale numerical model of lake-effect storms. J. Atmos. Sci. 29 (1972) 1025-1040.

[30] P.D. Lax and X.-D. Liu, Solution of two-dimensional Riemann problems of gas dynamics by positive schemes. SIAM J. Sci. Comput. 19 (1998) 319-340 (electronic).

[31] R.J. LeVeque, High resolution finite volume methods on arbitrary grids via wave propagation. J. Comput. Phys. 78 (1988) $36-63$.

[32] R.J. LeVeque, Wave propagation algorithms for multi-dimensional hyperbolic systems. J. Comput. Phys. 131 (1997) $327-353$.

[33] R.J. LeVeque, Finite Volume Methods for Hyperbolic Problems. Cambridge Texts in Applied Mathematics. Cambridge University Press, Cambridge (2002).

[34] D. Levy, G. Puppo and G. Russo, Central WENO schemes for hyperbolic systems of conservation laws. ESAIM: M2AN 33 (1999) 547-571.

[35] D. Levy, G. Puppo and G. Russo, Compact central WENO schemes for multidimensional conservation laws. SIAM J. Sci. Comput. 22 (2000) 656-672.

[36] D. Levy, G. Puppo and G. Russo, A fourth-order central WENO scheme for multidimensional hyperbolic systems of conservation laws. SIAM J. Sci. Comput. 24 (2002) 480-506.

[37] K.-A. Lie and S. Noelle, On the artificial compression method for second-order nonoscillatory central difference schemes for systems of conservation laws. SIAM J. Sci. Comput. 24 (2003) 1157-1174.

[38] R. Liska and B. Wendroff, Comparison of several difference schemes on 1D and 2D test problems for the euler equations. SIAM J. Sci. Comput. 25 (2003) 995-1017.

[39] W. Liu, J. Cheng and C.-W. Shu, High order conservative Lagrangian schemes with Lax-Wendroff type time discretization for the compressible Euler equations. J. Comput. Phys. 228 (2009) 8872-8891.

[40] M. Lukáčová-Medviďová, K.W. Morton and G. Warnecke, Finite volume evolution Galerkin methods for hyperbolic systems. SIAM J. Sci. Comput. 26 (2004) 1-30.

[41] J.P. McCreary, P.K. Kundu and R.L. Molinari, A numerical investigation of dynamics, thermodynamics and mixed-layer processes in the Indian Ocean. Prog. Oceanog. 31 (1993) 181-244.

[42] H. Nessyahu and E. Tadmor, Nonoscillatory central differencing for hyperbolic conservation laws. J. Comput. Phys. 87 (1990) 408-463.

[43] J. Panuelos, J. Wadsley and N. Kevlahan, Low shear diffusion central schemes for particle methods. J. Comput. Phys. 414 (2020) 109454.

[44] P. Ripa, On improving a one-layer ocean model with thermodynamics. J. Fluid Mech. 303 (1995) 169-201.

[45] P.L. Roe, Approximate Riemann solvers, parameter vectors, and difference schemes. J. Comput. Phys. 43 (1981) $357-372$.

[46] M.L. Salby, Deep circulations under simple classes of stratification. Tellus 41A (1989) 48-65.

[47] C.W. Schulz-Rinne, Classification of the Riemann problem for two-dimensional gas dynamics. SIAM J. Math. Anal. 24 (1993) $76-88$.

[48] C.W. Schulz-Rinne, J.P. Collins and H.M. Glaz, Numerical solution of the Riemann problem for two-dimensional gas dynamics. SIAM J. Sci. Comput. 14 (1993) 1394-1394.

[49] J. Shi, Y.-T. Zhang and C.-W. Shu, Resolution of high order WENO schemes for complicated flow structures. J. Comput. Phys. 186 (2003) 690-696.

[50] P.K. Sweby, High resolution schemes using flux limiters for hyperbolic conservation laws. SIAM J. Numer. Anal. 21 (1984) 995-1011.

[51] E.F. Toro, Riemann Solvers and Numerical Methods for Fluid Dynamics: A Practical Introduction, 3rd edition. Springer-Verlag, Berlin, Heidelberg (2009).

[52] B. van Leer, Towards the ultimate conservative difference scheme. V. A second-order sequel to Godunov's method. J. Comput. Phys. 32 (1979) 101-136.

[53] W.R. Young, The subinertial mixed layer approximation. J. Phys. Oceanogr. 24 (1994) 1812-1826.

[54] V. Zeitlin, Geophysical Fluid Dynamics: Understanding (almost) Everything with Rotating Shallow Water Models. Oxford University Press, Oxford (2018).

[55] Y. Zheng, Systems of conservation laws: Two-dimensional Riemann Problems. In: Vol. 38 of Progress in Nonlinear Differential Equations and their Applications. Birkhäuser Boston, Inc., Boston, MA (2001). 